\title{
In vitro Topical Delivery of Chlorhexidine to the Cornea: Enhancement using Drug-Loaded Contact Lenses and $\beta$-Cyclodextrin Complexation, and the Importance of Simulating Tear Irrigation
}

Melissa G. Hewitt ${ }^{\mathrm{a}}$, Peter W. J. Morrison ${ }^{\mathrm{a}, \mathrm{b}}$, Hannah M. Boostrom ${ }^{\mathrm{a}}$, Siân R. Morgan ${ }^{\mathrm{a}, \mathrm{b}}$, Melissa Fallon $^{\mathrm{a}, \mathrm{b}}$, Philip N. Lewis ${ }^{\mathrm{a}, \mathrm{b}}$, David Whitaker ${ }^{\mathrm{b}}$, Andrea Brancale ${ }^{\mathrm{a}}$, Carmine Varricchio $^{\mathrm{a}}$, Andrew J. Quantock ${ }^{\mathrm{b}}$, Matthew J. Burton ${ }^{\mathrm{c}, \mathrm{d}}$, Charles M. Heard ${ }^{\mathrm{a}}$

${ }^{\text {a }}$ School of Pharmacy and Pharmaceutical Sciences, Cardiff University, Wales, UK. CF10 $3 \mathrm{NB}$

${ }^{\mathrm{b}}$ School of Optometry and Vision Sciences, Cardiff University, Wales, UK. CF24 4HQ

${ }^{\mathrm{c}}$ International Centre for Eye Health, Faculty of Infectious \& Tropical Diseases, London School of Hygiene and Tropical Medicine, London WC1E 7HT

${ }^{\mathrm{d}}$ Moorfields Eye Hospital NHS Foundation Trust, London

Correspondence. Charles M Heard School of Pharmacy and Pharmaceutical Sciences, Cardiff University, Wales, UK. CF10 3NB

Tel +44 (0)2920 875819

Email. heard@cardiff.ac.uk 


\begin{abstract}
Microbial keratitis is a severe, sight threatening condition caused by various pathogens. Eyedrops are the standard delivery modality for treating these disorders; however, blinking reflex, elevated tear production and nasolacrimal drainage eliminate much of the instilled dose within a few seconds. Therefore, eye drops must be applied repeatedly for prolonged periods. The present study aimed to probe more effective ocular delivery of chlorhexidine based upon drug-loaded hydrogel contact lenses and $\beta$-cyclodextrin $(\beta-\mathrm{CD})$, whilst also determining the effect of constant irrigation with simulated tear fluid (STF) in in vitro experiments.
\end{abstract}

Chlorhexidine digluconate (as 0.2 and $2.0 \%$ solution, $\beta-\mathrm{CD}$ inclusion complexes and loaded hydrogel contact lenses) were applied to enucleated porcine eyes as single or multiple $10 \mu \mathrm{L}$ doses, or as drug-loaded contact lenses, with and without $\beta$-CD. The corneas were then excised and drug extracted quantified by HPLC. The effect of constant irrigation by STF was evaluated to test the effect of increased tear production on corneal delivery. Potential antimicrobial activity of delivered drug was also assessed.

Results showed that drug-loaded contact lenses delivered the greatest amount of chlorhexidine into the cornea over a $24 \mathrm{~h}$ period, whilst the eyedrop solution comparator delivered the least. The $\beta$-CD significantly enhanced chlorhexidine delivery to the cornea from eyedrop solution, although contact lenses loaded with chlorhexidine- $\beta$ - $C D$ failed to enhance delivery. $\beta$-CD within the hydrogel matrix impeded drug release. Constant irrigation with STF significantly reduced the amount of drug delivered to the cornea in all cases. Chlorhexidine retained antimicrobial activity in all delivery methods.

Hydrogel contact lenses loaded with chlorhexidine delivered significantly higher levels to the cornea compared to eyedrops, either multiple hourly doses or a single dose. They also offer reduced application, in particular to a non-ulcerated corneal infection. Finally, the importance of fully accounting for tear production in in vitro ocular delivery experiments was highlighted.

KEYWORDS Antimicrobial, $\beta$-cyclodextrin, chlorhexidine, contact lens, cornea, E. coli, hydrogel, keratitis, NMR. 


\section{Introduction}

Microbial keratitis (MK) is a condition in which the cornea becomes inflamed due to the presence of pathogens and, if not promptly treated, can cause permanent vision damage or even total blindness. ${ }^{1}$ It is a particular problem in low and middle-income countries, where it has been reported as the second most common cause of corneal blindness ${ }^{2}$, for example, in sub-Saharan Africa corneal scarring, mostly from keratitis, accounts for $15 \%$ of monocular blindness; and is also problematic in other animals. ${ }^{3} \mathrm{MK}$ is usually caused by infection with pathogenic microorganisms gaining access to the stroma following epithelial trauma such as abrasions or improper contact lens use, where they subsequently proliferate, triggering a host inflammatory response and further symptoms including severe pain, blurred vision, photosensitivity, increased tear production and typically ulceration. ${ }^{4,5}$ Invasive microorganisms can include bacteria, viruses, fungi and protozoans in particular Acanthamoeba. Patients presenting with bacterial keratitis typically respond well to current treatments, although cases caused by fungi (including Fusarium and Aspergillus) and Acanthamoeba, tend to have poorer responses. The reasons for this are multifactorial but can include ineffectual drug delivery systems. ${ }^{5,6}$ Therapeutic surgical intervention, including penetrating keratoplasty is required in the severest cases which are unresponsive to drug therapy. ${ }^{1,7-9}$

Chlorhexidine digluconate (Figure 1) is a widely used broad-spectrum microbicidal agent ${ }^{10-12}$ that has previously been proposed for treating fungal ${ }^{13}$ and Acanthamoeba keratitis. ${ }^{14-16}$ Chlorhexidine can attach to the cellular membranes of microbes and disrupt their integrity, penetrate cells and cause leakage of their components and ultimately cell death. ${ }^{17,18}$ Typically, current treatments are delivered in the form of topically applied conventional eyedrops, which have the advantage of being inexpensive and easily administered. However, whilst the cornea may appear to be easily accessible for treatment, delivery into this tissue is particularly challenging with numerous physicochemical barriers hindering drug penetration. Additionally, other factors such as elevated tear production, blinking reflex and nasolacrimal drainage work together to significantly reduce ocular residence time of a drug, such that only 1 to $7 \%$ of the instilled drug reaching the stroma. In practical terms, eye drops must thus be applied frequently, usually initially hourly or half hourly for several days, and then gradually tailed off over several weeks to maintain a therapeutic concentration in the cornea. ${ }^{19-21}$ Such demanding administration regimens can result in poor patient compliance and discontinuation of treatment, whilst also potentially 
increasing the risk of toxicity. ${ }^{22,23}$ Additionally, nasolacrimal drainage can result in systemic absorption of the drug, potentially leading to adverse side effects. ${ }^{24}$ For the relatively low amount of drug that remains, penetration of the corneal epithelium is challenging due to the diffusional barriers ${ }^{25}$ and due to low concentration. As is the case for most membranes, drug permeation across intact corneal barriers is governed by Fick's first law:

$$
J=-D \frac{d C}{d x}
$$

Where $\mathrm{J}$ is flux $\left[\mathrm{cm}^{-2} / \mathrm{s}\right], \mathrm{D}$ is diffusivity $\left[\mathrm{cm}^{2} / \mathrm{s}\right]$ and $\delta \mathrm{C} / \delta \mathrm{x}$ is the concentration gradient $\left[\mathrm{cm}^{-}\right.$ $\left.{ }^{4}\right]$. The flux of a molecule across a membrane is thus enhanced when: 1. the concentration gradient is high (Figure 2), achievable if the dose can be retained at the cornea surface, ${ }^{26}$ and/or 2. the membrane is modulated such that its diffusivity is increased. These two parameters may also be used cooperatively to achieve even greater rates of drug delivery.

Generally used to increase the concentration of a poorly water-soluble drug in an aqueous solution, cyclodextrins (CD) are cyclic oligosaccharides composed of D-glucose units, linked by $\alpha-1,4$-glucosidic linkages (Figure 1). This is exploited in ocular drug delivery, as drug-cyclodextrin inclusion complexes have improved solubility, reduce irritation and enhance drug permeability. ${ }^{27}$ Chlorhexidine has been shown to form inclusion complexes with $\beta$-cyclodextrin $(\beta-\mathrm{CD})^{28,29}$ resulting in enhanced uptake and release properties from polymeric nanogels. ${ }^{30}$ Drug-loaded soft hydrogel contact lenses are an attractive and convenient ocular drug delivery approach. Consisting of a three-dimensional polymer network, contact lenses possess the ability to absorb drug solutions and retain them in their hydrogel matrix. ${ }^{31}$ By attaching to the tear film over the cornea, they increase the ocular residence time of the incorporated medication and, thus, allow greater opportunity for drug release and instillation. ${ }^{26}$ Sustained, controlled release of the entrapped drug is also achieved via passive diffusion out of the hydrogel matrix of lenses and into the cornea, as according to Fick's diffusion law, telling us that the greater the concentration gradient, the great the drug flux rate (Figure 2). This is important as microbial infiltrates are generally located within the aqueous environment of the stroma, located between the epithelial membrane and Bowman's layer of the cornea. Also, the extended residence time of contact lenses can circumvent the frequent dosing issues associated with eyedrops and produce higher drug penetration. ${ }^{32}$

Furthermore, challenges arise in the field of ocular formulation development when considering how to test formulations. In vitro or ex vivo experiments using excised porcine or 
bovine eyes are typical, yet such experiments need to be representative of in vivo ocular delivery. When used in static model, tear production and blinking are typically not accounted for. An additional aim of this work was therefore to investigate the effect of constant dropwise irrigation of the eye with simulated tear fluid (STF) on ocular drug delivery. We then used this model to probe the enhanced ocular delivery of chlorhexidine into porcine corneas in vitro using a combination of $\beta-\mathrm{CD}$ and hydrogel contact lenses for enhanced delivery of chlorhexidine to the cornea as a prospective treatment for stromal keratitis.

\section{Experimental}

\subsection{Materials}

Chlorhexidine digluconate (20\% solution), LB agar, phosphate buffer saline (PBS) tablets, $\mathrm{NaCl}, \mathrm{NaHCO}_{3}, \mathrm{CaCl}_{2}$ and $\beta$-cyclodextrin $(\beta-\mathrm{CD})$ were purchased from Sigma-Aldrich, Gillingham, UK. KCl was purchased from Fisher Scientific, Loughborough, UK. Freshly excised porcine eyes were obtained from a local abattoir on the day of slaughter and kept in a refrigerator overnight for use the next day. Clariti ${ }^{\mathrm{TM}} 1$ Day silicone hydrogel contact lenses were a gift from Cardiff University Eye Clinic. Escherichia coli, National Collection of Type Cultures (NCTC) strain reference 10418 was purchased from NCTC, Salisbury, UK.

\subsection{Preparation of solutions and drug loaded contact lenses}

Chlorhexidine digluconate, $20 \%$, was diluted with deionised water to obtain $0.2 \%, 2.0 \%$ solutions - lower levels were not evaluated in this work. To test the effect of including cyclodextrin to the eyedrop solution, $30 \mathrm{mg} / \mathrm{mL} \beta-\mathrm{CD}$ was added to $2.0 \%$ chlorhexidine and stirred overnight at $2-4{ }^{\circ} \mathrm{C}$. Clariti ${ }^{\mathrm{TM}} 1$-day silicone hydrogel contact lenses were soaked in $10 \mathrm{~mL}$ deionized water for $4 \mathrm{~h}$ on a rocking plate to remove storage medium, before being immersed in test solutions overnight using a tube rotator. Prior to use the lenses were removed from solution, lightly blotted and weighed.

\subsection{Preparation and use of simulated tear fluid (STF)}

As we were investigating dilution/wash-out effects an electrolyte-only STF was used, prepared based upon a previously reported method. ${ }^{33} \mathrm{In}$ a glass beaker $0.68 \mathrm{~g} \mathrm{NaCl}, 0.22 \mathrm{~g}$ 
$\mathrm{NaHCO}_{3}, 0.008 \mathrm{~g} \mathrm{CaCl}_{2}, 0.14 \mathrm{~g} \mathrm{KCl}$ were dissolved in 1L deionized water. STF was delivered to the corneas of excised porcine eyes using capillary tubing and a gravity-fed reservoir at a continuous and reproducible rate of $100 \mu \mathrm{L} / \mathrm{min}$, maintaining an evenly distributed artificial tear film on in vitro eyes for the duration of the experiments. To directly test the effect of STF, an equal number of eyes received constant irrigation, the other half remained non-irrigated.

\subsection{In vitro corneal penetration}

Enucleated porcine eyes were checked for abrasions with a magnifying glass, before being trimmed of excess tissue and placed cornea uppermost into the wells of a 6-well plate; each well containing $2 \mathrm{~mL}$ PBS at the bottom of the well to limit dehydration with corneas well above this level. The plate with eyeballs was placed in a water bath set at $37^{\circ} \mathrm{C}$ before being dosed with drug treatment. Using a calibrated pipette, $10 \mu \mathrm{L}$ aliquots of eyedrop solution were applied - an amount comparable to the residual volume remaining after delivery with commercial eyedrops (around $30 \mu \mathrm{L}$ ) after a single blink, therefore simulating an in vivo scenario. $^{34}$

Single dose: the eyes were dosed with a single $10 \mu \mathrm{L}$ aliquot of 0.2 or 2.0 chlorhexidine digluconate eye drop or a contact lens soaked in the corresponding solution to determine the effect of concentration on delivery. To determine the effect of $\beta-C D$ addition, eyes were dosed once with $10 \mu \mathrm{L}$ eyedrops of $2.0 \%$ chlorhexidine, $2.0 \%$ chlorhexidine in combination with $30 \mathrm{mg} / \mathrm{mL} \beta-\mathrm{CD}$ or contact lenses containing $2.0 \%$ chlorhexidine or $2.0 \%$ chlorhexidine $+30 \mathrm{mg} / \mathrm{mL} \beta$-CD. The eyes were left for $2 \mathrm{~h}, 6 \mathrm{~h}$ or overnight (approximately $24 \mathrm{~h}$ ), with half the eyes receiving continuous irrigation with STF at a rate of $0.1 \mathrm{~mL} / \mathrm{min}$ to mimic tear

Multiple dose: to simulate current clinical practice in the ocular administration of chlorhexidine, porcine eyes were dosed with $10 \mu \mathrm{L}$ aliquots of 0.2 or $2.0 \%$ chlorhexidine digluconate at $\mathrm{T}_{0}$ and then at hourly intervals up to a total of 6 hours ( 7 doses) and all under constant STF irrigation (simulated in vivo scenario). At 1 hour after the final dose the corneas were excised. As a comparison, contact lenses soaked in chlorhexidine solution were applied for the same durations.

\subsection{Cornea extraction}


After the predetermined time, corneas were excised by blunt dissection using a scalpel where contact lenses were used, any residual chlorhexidine was first removed from the ocular surface by further irrigation with STF. The excised corneas had a wet-weight of $0.2269 \pm$ $0.0201 \mathrm{~g}(\mathrm{n}=6, \pm \mathrm{SD})$; any that were found to be out of this range were not used. The corneas were then cut into small pieces and chlorhexidine extracted by gentle mixing in two sequential volumes of methanol for $18-24 \mathrm{~h}$ each. The methanol from the both extraction steps was combined, centrifuged for $15 \mathrm{~min}$ and supernatants evaporated at $60{ }^{\circ} \mathrm{C}$ overnight. Residues were reconstituted with $1 \mathrm{~mL}$ mobile phase and transferred to autosampler vials for HPLC analysis.

\subsection{High Performance Liquid Chromatography (HPLC) analysis}

Chlorhexidine was determined by HPLC analysis using an Agilent 1100 system comprised of a G1379B degasser, G1311A quaternary pump, G1313A ALS autosampler and G1314A VWD detector. The separation method employed an isocratic mobile phase of 50:50 v/v acetonitrile:5 \% aqueous trifluoracetic acid and a Phenomenex Kinetex $5 \mu \mathrm{m}$ C18 150 x 4.6 $\mathrm{mm}$ column. The detection wavelength was set at $245 \mathrm{~nm}$, injection volume $20 \mu \mathrm{L}$ and flow rate $1.5 \mathrm{~mL} / \mathrm{min}$. Under these conditions, chlorhexidine was found to have a retention time of approximately $1.2 \mathrm{~min}$. A chlorhexidine calibration curve was constructed in mobile phase over the concentrations 1.00 to $7.81 \times 10^{-3} \mathrm{mg} / \mathrm{mL}$. The absence of potential matrix effects a calibration curve prepared in aqueous centrifuged and filtered corneal homogenate provided the same calibration coefficient.

2.7 NMR and molecular modeling analysis of chlorhexidine- $\beta$-cyclodextrin inclusion complexation

NMR spectra were recorded on a Bruker AVANCE 500 spectrometer (500 MHz) and autocalibrated to the deuterated solvent reference peak. Chemical shifts are given in $\delta$ relative to tetramethylsilane (TMS); the coupling constants (J) are given in Hertz. TMS was used as an internal standard $(\delta=0)$ for ${ }^{1} \mathrm{H}-\mathrm{NMR}$ and $\mathrm{CDCl}_{3}$ served as an internal standard $(\delta=77.0)$ for 13C-NMR. The chlorhexidine $20 \%$ solution in water and chlorhexidine- $\beta$-CD samples were analyzed suppressing water solvent peak.

\subsection{Molecular modeling}


Computational studies were carried out on a $1.80 \mathrm{GHz}$ Intel Xeon ( 8 cores) processor-based computer, running Ubuntu 14.04 LTS. Preparation of the structure, simulations and analysis were carried out with Maestro v 11.4, (Schrödinger LLC, New York, NY, 2017). The molecular dynamic (MD) simulations were performed using the Desmond package (Schrödinger New York, NY, 2017). The initial chlorhexidine- $\beta$-CD complex was manually prepared following the structural information reported previously. ${ }^{35}$ The $\beta$-CD complex was solvated with TIP3P water such that a water shell $10 \AA$ in thickness was formed around the complex, using OPLS3 force field. The solvated complex was then simulated with the NPT relax protocol in Desmond. The protocol involved an initial minimization of the solvent with restrains on the solute, which was followed by MD simulations of $500 \mathrm{~ns}$ in NPT ensembles with temperature $(300 \mathrm{~K})$ and pressure (1 atm) constant. Data were collected every $100 \mathrm{ps}$ (trajectory). Each chlorhexidine- $\beta$-CD complex simulation was performed in triplicate, using a random seed as a starting point each time. The estimated $\Delta \mathrm{G}_{\text {binding }}$ was calculated using the Desmond command-line script thermal_mmgbsa.py collecting the value every 100 ps and a stability constant was determined from a standard phase solubility plot. Then, van der Waals volume predictions of chlorhexidine and chlorhexidine- $\beta-\mathrm{CD}$ complex ( VvdW) were carried out with MOE 2015.10, where the van der Waals volume is defined as the space occupied by the molecule, which is impenetrable to other molecules at standard conditions.

\subsection{Antimicrobial well diffusion assay}

A modified version of the well diffusion assay was performed to demonstrate that the chlorhexidine delivered to corneas was antimicrobially active against $E$. coli-a model organism chosen to demonstrate bacterial keratitis. ${ }^{36}$ In triplicate, porcine eyes were treated as previously described with either a $2.0 \%$ chlorhexidine eyedrop, a $2.0 \%$ chlorhexidine + $30 \mathrm{mg} / \mathrm{mL} \beta$-CD eyedrop, a $2.0 \%$ chlorhexidine-soaked contact lens, a $2.0 \%$ chlorhexidine + $30 \mathrm{mg} / \mathrm{mL} \beta$-CD soaked contact lens, or control eyedrops or contact lenses (deionized water), for $6 \mathrm{~h}$ with STF irrigation before corneal excision. LB agar was prepared from dry powder according to manufacturer's instructions and poured to a depth of $4 \mathrm{~mm}$ in $100 \mathrm{x} 15 \mathrm{~mm}$ petri dishes. E. coli was revived from glycerol stock previously prepared from NCTC-sourced ampoule and frozen at $-80{ }^{\circ} \mathrm{C}$. A 0.5 MacFarland suspension of E. coli was prepared in PBS by adjusting the optical density to $0.1 \pm 0.2 \mathrm{AU}$ at $625 \mathrm{~nm}$. Cotton swabs were used to spread suspension evenly over the surface of LB agar plates before using a sterile $15 \mathrm{~mm}$ diameter cork borer to remove a disk of agar from the center, creating a well in each plate. A single 
excised cornea was placed in each agar well and covered with $300 \mu \mathrm{L}$ PBS before incubation at $37^{\circ} \mathrm{C}$ for $18-24 \mathrm{~h}$. The diameter of the zone of growth inhibition was measured in $\mathrm{mm}$.

\subsection{Statistical Analysis}

Statistical analysis was performed using InStat statistical package (GraphPad Software Inc. San Diego, CA, USA). ANOVA was performed on all data sets to assess for significant differences in corneal chlorhexidine levels between delivery methods and to compare the zone of inhibitions from the well diffusion assay, where $p<0.05$ was considered significant.

\section{Results}

3.1 Single dose: delivery of chlorhexidine to the cornea

The time course for the amount of chlorhexidine delivered into the cornea was determined at 2, 6 or 24 h. Eyes were dosed with $2.0 \%$ chlorhexidine eyedrops or $2.0 \%$ soaked contact lenses, both with or without the addition of $\beta-C D$, and were incubated with or without STF irrigation. The highest amount of chlorhexidine was delivered to the cornea from chlorhexidine-only contact lenses (blue square) at all time points $(p<0.001)$, with eyedrops delivering $0.43 \pm 0.6 \mu \mathrm{g}$ and $5.13 \pm 2.1 \mu \mathrm{g}$ chlorhexidine to the cornea (with and without STF, respectively) and the contact lenses delivering over sixty times the amount of chlorhexidine with $(26.1 \pm 6.16 \mu \mathrm{g})$ and over seven times without STF $(36.25 \pm 9.43 \mu \mathrm{g})$ compared to the eyedrops after $6 \mathrm{~h}$ (Figure 3 ). Addition of $\beta-\mathrm{CD}$ to eyedrops significantly ( $p$ $<0.001$ ) increased the amount of chlorhexidine delivered to the cornea at all time points when compared to standard eyedrops, \pm STF. For example, at $2 \mathrm{~h}$ with STF, only $1.84 \pm 0.23 \mu \mathrm{g}$ was delivered with the standard eyedrops but over six times the amount, $11.71 \pm 3.0 \mu \mathrm{g}$, was delivered when $\beta$-CD was added. In addition, after $6 \mathrm{~h}$, a low $0.43 \pm 0.6 \mu \mathrm{g}$ chlorhexidine remained in the cornea when delivered with the standard eyedrops, but a 19-fold increase in chlorhexidine was observed when $\beta-\mathrm{CD}$ was included in the eyedrops without STF.

Chlorhexidine-loaded contact lenses, however, still delivered significantly $(p<0.001)$ more chlorhexidine to the cornea than chlorhexidine- $\beta-C D$ eyedrops, at all time points with and without the addition of STF (Figure 3). However, addition of $\beta-\mathrm{CD}$ to the drug loaded contact lenses did not improve chlorhexidine delivery when compared to the chlorhexidineonly lenses. At all timepoints, with or without the addition of STF, no significant difference $(p>0.05)$ in the amount of drug delivered to the cornea was found between standard and $\beta$ - 
CD contact lenses. In fact, it can be seen that the contact lenses delivered slightly more drug at all time intervals without $\beta$-CD being added (under the same STF conditions).

Although not statistically significant, when delivering chlorhexidine from contact lenses without STF, an increase in the amount of drug reaching the cornea was indicated between 2 and $6 \mathrm{~h}$ time points, whereas the amount decreased by $24 \mathrm{~h}$ (Figure 3). When irrigated with STF, there was also an increase in chlorhexidine delivered to the cornea between 2 and $6 \mathrm{~h}$ from the contact lenses, but less so than without STF, and this likewise decreased after $24 \mathrm{~h}$.

The chlorhexidine- $\beta$-CD contact lenses applied to eyes not receiving STF also produced a significant $(p<0.01)$ increase in corneal chlorhexidine from 2 to $6 \mathrm{~h}$, then a decrease in drug delivered at $24 \mathrm{~h}$. With the addition of STF, this effect was negligible with the levels of chlorhexidine remaining fairly similar throughout the time intervals. While the amount of chlorhexidine delivered from eyedrops did not change as markedly over time as with contact lenses, a small but insignificant increase in the amount delivered between 2 and $6 \mathrm{~h}$ was observed without STF whereas with STF the amount of chlorhexidine delivered to the cornea decreased over time. These data demonstrate the effect of STF on quantification of ocular drug delivery. Constant ocular irrigation clearly reduces the amount of chlorhexidine absorbed into the cornea in every delivery scenario, the greatest difference being observed with the $\beta$-CD contact lenses at $6 \mathrm{~h}$ (Figure 3 ).

\subsection{Multiple $1 \mathrm{~h}$ doses of eyedrops vs contact lenses, with STF irrigation}

Table 1 shows the amount of chlorhexidine localised in porcine corneas as a function of dosing frequency (hourly), with extraction of corneas 1 hour after final dose. The experiments were conducted under constant irrigation with STF to simulate in vivo conditions. Two drug concentrations were used, $2 \%$ and $0.2 \%$ - the latter was intended to simulate a typical regimen currently used to treat fungal keratitis.

When dosed with $0.2 \%$, the chlorhexidine reached steady state after 5 hours following the instillation of 4 x $10 \mu \mathrm{L}$ drops. Steady state was attained at a dose of $\sim 1 \mu \mathrm{g}$. By comparison, the chlorhexidine level continued to rise over the duration of the experiment, when contact lenses loaded with $0.2 \%$ chlorhexidine were used. After 7 hours, the $0.2 \%$ drop provided $1.01 \pm 0.25 \mu \mathrm{g}$ chlorhexidine, whereas the lens provided 10-fold this amount at 10.9 $\pm 0.61 \mu \mathrm{g}(p<0.05)$. The total amount of chlorhexidine dosed over the course of this experiment was $120 \mu \mathrm{g}$. 
When dosed with $2 \%$ chlorhexidine drops, steady state was achieved after 4 hours (3 $\mathrm{x} 10 \mu \mathrm{L}$ drops), at a level of $\sim 6 \mu \mathrm{g}$. This is $6 \mathrm{x}$ more that achieved from $0.2 \%$ drops $(p<0.05)$. Using $2 \%$ loaded contact lenses the concentration seemed to reach steady state after 6 hours, at a level of approximately $26 \mu \mathrm{g}$ per cornea. This was $\sim 4.2 \mathrm{x}$ the level obtained with $2 \%$ chlorhexidine drops $(p<0.05)$. Furthermore, the value of $26 \mu \mathrm{g}$ matches very closely the value of $26 \mu \mathrm{g}$ obtained in 3.1 for the single dose at 6 hours. The total amount of chlorhexidine dosed onto the eyes was $1200 \mu \mathrm{g}$.

\subsection{NMR \& molecular modelling}

NMR analysis was performed in order to examine the formation of chlorhexidine- $\beta-C D$ complexation. The chlorhexidine spectrum showed two broad doublets in the aromatic region $(\delta 7.0$ and $6.9 \mathrm{ppm})$, and three peaks for the protons in the aliphatic biguanide internal chain ( $\delta 2.82,1.12$ and $0.89 \mathrm{ppm})$. The comparison between chlorhexidine and chlorhexidine- $\beta-\mathrm{CD}$ clearly showed a change in the aromatic protons H8, H7, H11 and H12 (Figure 4) of the phenyl moiety, that are slightly deshielded in the chlorhexidine- $\beta-C D$ complex $(\delta 7.28$ and $7.13 \mathrm{ppm}, \delta$ of $+0.2 \mathrm{ppm})$. This de-shielding effect suggests the proximity of electronegative atoms (e.g. oxygens of cyclodextrin) to the aromatic protons, and this change in electron density increased the chemical shift of the aromatic protons. These observations might indicate that chlorhexidine aromatic rings are inserted in the $\beta-\mathrm{CD}$ cavity and are consistent with those described in the literature. ${ }^{37,38}$ Molecular modelling studies were conducted to further interpret the NMR data. As shown in these earlier papers, which included Job's plot analysis, a 1:2 ratio complex of chlorhexidine to $\beta-\mathrm{CD}$ was also found possible in this simulation, with the inclusion of the aromatic rings of the drug in the less polar but more lipophilic central cavity of the $\beta-\mathrm{CD}$. This inclusion complexation is stabilized through van der Waals and hydrophobic forces. Figure 5 shows space-filling depictions of chlorhexidine and chlorhexidine- $\beta-C D$ inclusion complex involving two $\beta-C D$ molecules, allowing the comparison of molecular volumes of $477.13 \AA 3$ /molecule and $2283.12 \AA 3 /$ complex respectively.

In order to better understand the inclusion of chlorhexidine in $\beta-\mathrm{CD}$, a series of $500 \mathrm{~ns}$ dynamics simulations were performed evaluating the change in van der Waals energy in the system composed by chlorhexidine and two $\beta$-CD molecules. The variations of van der Waals energy during such dynamic simulations can be considered a significant parameter in the prediction of host-guest inclusion complex stability, with high energy indicating a less favorable - and therefore less probable - conformation. In the current investigation, the 
position and orientation of the guest molecule with respect to the host was mainly perpendicular with the two mono-chlorophenyl rings inside the cavity. While this arrangement showed the lowest energy during the entire MD simulation (Figure 6), the complex was not static, but rather continuously forming and dissociating during the simulation. This observation was supported by the binding free energy calculations, confirming that the van der Waals interaction was the key force in forming the complexes, and moreover showing the high binding affinity of chlorhexidine in complex with $\beta-\mathrm{CD}$ at a 1:2 ratio ( $\Delta \mathrm{G}$ average $-53.56 \pm$ Standard Deviation 27.50) as shown in Figure 7. The difference in binding energy ( $\Delta \mathrm{G}$ Range: -87.67 to 1.24 ) can explain the desolvation process and release of chlorhexidine from the $\beta-\mathrm{CD}$ carrier system. This is supported by a relatively low stability constant $(\mathrm{Kc})$ of value $\left(201.92 \mathrm{M}^{-1}\right)$ as determined using the Higuchi \& Connors $(1965)^{39}$ approach (data not shown) which is probably due to the presence of the 2 positive charges. ${ }^{40}$ Lying within the range $100-1000 \mathrm{M}^{-1}$, this value suggests that the chlorhexidineHP- $\beta$-cyclodextrin complex is sufficiently stable within the aqueous solution, yet the interaction between drug and carrier is weak allowing dissociation ${ }^{41}$, as indicated in Figure 7, allowing the drug to penetrate the cornea.

In summary, in silico modelling showed that masking the lipophilic aromatic rings of chlorhexidine inside the $\beta$-CD molecules could allow the chlorhexidine molecule to approach the corneal epithelium more effectively, and dissociation/release thereafter making chlorhexidine available to penetrate into the cornea.

\subsection{Contact lenses}

A mean of $794.3 \pm 37.05 \mu \mathrm{g}$ chlorhexidine was loaded into the chlorhexidine-loaded lenses, whilst $773.9 \pm 97.3 \mu \mathrm{g}$ chlorhexidine was loaded into the chlorhexidine- $\beta$-CD lenses. There was no significant difference between chlorhexidine loading with and without $\beta-\mathrm{CD}$ ( $p>0.05)$. Contact lenses containing chlorhexidine only delivered a higher percentage of their initial dose to the cornea compared to the chlorhexidine eyedrops at all time points $(p$ $<0.001$ ), with $2.39 \pm 0.9 \%$ delivered at $2 \mathrm{~h}, 4.32 \pm 1.2 \%$, at $6 \mathrm{~h}$ and $1.44 \pm 0.01 \%$ after $24 \mathrm{~h}$. Addition of $\beta-C D$, in accordance with the previous findings, did not increase the percentage of drug delivered from the initial dose in the contact lenses. Unlike the chlorhexidine and chlorhexidine- $\beta-C D$ eyedrops, equal percentages were delivered after $2 \mathrm{~h}(2.39 \%)$, and significantly less $(p<0.001)$ was delivered after 6 and $24 \mathrm{~h}$ from the $\beta$-CD contact lens $(1.87$ $\pm 0.69 \%$ and $1.18 \pm 0.71 \%$, respectively). 
These figures are low compared to previous research, for example, it was reported that at least $20 \%$ of the timolol entrapped within their soft gel contact lens entered the cornea, which was much larger than the fractional uptake for drug delivery through eyedrops. ${ }^{22}$ However, different chemistries in terms of both lens polymer and loaded drugs make extrapolations and comparisons to other systems unrealistic.

\subsection{Antimicrobial diffusion assay}

With a reported chlorhexidine minimum inhibitory concentration (MIC) of $4 \mu \mathrm{g} / \mathrm{mL}^{42}$ E. coli NCTC 10418 was selected as a susceptible model organism to confirm delivery of active drug to porcine corneas after $6 \mathrm{~h}$. The zones of inhibition produced by corneas treated with chlorhexidine eyedrops and contact lenses with and without $\beta$-CD can be seen in Figure 8 and Table 2.

Corneas treated with contact lenses containing only chlorhexidine exhibited the largest zone of inhibition of all conditions tested $(30.3 \pm 1.0 \mathrm{~mm})$, whilst chlorhexidine-only eyedrops delivered the least active drug to corneas, producing the smallest observed zone of inhibition of $18.3 \pm 2.9 \mathrm{~mm}$ (note: this included the diameter of the well which was $15 \mathrm{~mm}$ ). When treated using chlorhexidine- $\beta-\mathrm{CD}$ contact lenses, wells containing the excised cornea produced a zone of inhibition of $27.8 \pm 0.8 \mathrm{~mm}$ compared to $30.3 \pm 1.0 \mathrm{~mm}$ with the chlorhexidine-only contact lenses, as seen in Figure 7. Although this difference was not statistically significant $(p>0.05)$, the trend agrees with the previous findings that addition of $\beta-\mathrm{CD}$ to the contact lenses did not enhance chlorhexidine delivery but possibly reduced it slightly. Alternatively, less chlorhexidine was loaded into the chlorhexidine- $\beta$-CD contact lenses - the reduced effectiveness of such lenses may simply reflect this. However, there was no significant difference between the amounts of chlorhexidine loaded into the contact lenses so this cannot be assumed.

The increase in chlorhexidine delivered from eye drops when combined with $\beta-C D$ was reflected in the observed zones of inhibition with corneas receiving the chlorhexidine- $\beta$ $\mathrm{CD}$ eyedrops which produced a larger zone of inhibition than chlorhexidine-only eyedrops $(20.3 \pm 1.9 \mathrm{~mm}$ and $18.3 \pm 2.9 \mathrm{~mm}$, respectively). Although the difference between treatments was not statistically significantly, the zone produced by chlorhexidine- $\beta-C D$ eyedrop treated corneas was significantly larger $(p<0.01)$ than that produced by those given control eyedrops treatment $(0 \mathrm{~mm})$, whilst chlorhexidine-only eyedrops failed to deliver enough to the cornea to create a large enough zone of inhibition to be statistically different from the control $(p>0.05)$. 
By way of bridging the amount of drug delivered to the cornea from the various formulations with the observed microbiological effect, a plot of drug mass per cornea $(\mu \mathrm{g})$ versus inhibition zone $\left(\mathrm{mm}^{2}\right.$ ) was prepared (Figure 9); the regression showed a $\mathrm{R}^{2}$ of 0.92 .

\section{Discussion}

Topical ocular therapies delivered in the form of eyedrops generally have poor availability due to blinking, tear production and nasolacrimal drainage, meaning up to $95 \%$ of the drug in the applied dose is lost due to lacrimal washing or absorption through the conjunctiva., ${ }^{5,22}$ In the case of using chlorhexidine to treat keratitis, the eyedrop regimen is intensive, as explained. Moreover, it is commonplace for a patient to need to strictly apply eyedrops frequently for several weeks in order to maintain therapeutic corneal concentrations and allow the infection to clear. This can result in poor patient compliance and discontinuation of treatment, ultimately failing to resolve the infection. ${ }^{21}$ In an attempt to address these issues, we investigated an alternative delivery method including $\beta-\mathrm{CD}$ and drug loaded hydrogel contact lenses for the enhanced corneal delivery of chlorhexidine. It was further hypothesized that these two parameters may also be used cooperatively to achieve even greater rates of drug delivery.

\subsection{Effect of continual irrigation with STF}

A significant finding from this study was the marked effect of a simple electrolyte-based STF on the delivery of chlorhexidine to the cornea in vitro: irrespective of the delivery method STF consistently reduced the amount of drug delivered. A symptom of keratitis patients is an increased rate of tear production. Although many other chemicals are present, tears are almost entirely comprised of water. In the current work we were interested primarily in the dilution/washing away effects of tear fluid, hence a simple electrolyte-based STF was used based upon a previously reported method. ${ }^{33}$

Simulating in vivo conditions, STF would wash away and dilute the applied chlorhexidine before it can be fully absorbed through the corneal surface. This would be compounded by corneal reflex or blink reflex which would act to eliminate the liquid dose from the corneal surface, the greater the number of reflex blinks the greater the amount of 
drug washed away. The volume of the 'drop' delivered to the eye is also important; a larger drop would be expected to deliver more drug, but at the same time could elicit more reflex blinking. Nevertheless, findings highlight the potential overestimation errors that may occur in static in vitro corneal penetration experiments. In addition to washing away drug, the tear film presents an additional diffusional barrier which becomes more important with increasing drug lipophilicity. ${ }^{43}$ Chlorhexidine in its charged form is very polar and therefore likely to be washed away relatively readily, and perhaps even leached back out of the epithelium. The chlorhexidine delivery profiles in Figure 3 illustrate that application of STF prevented drug build up in the cornea due to wash out and/or leaching. This is particularly apparent when comparing the effect of STF on chlorhexidine delivered per cornea by eyedrops both with (CD) and without (D) $\beta$-CD. Lack of build-up while using STF indicates that the concentration gradient and driving force are weak and serves to highlight wash-out effects likely to be seen in vivo.

Under normal conditions human tear flow is $\sim 2 \mu \mathrm{L} / \mathrm{min}$, with an estimated residual volume of $7 \mu \mathrm{L},{ }^{19}$ although tear production rate is significantly elevated during corneal infection. ${ }^{44,45}$ In our irrigation model, the STF was delivered at a rate $\sim 100 \mu \mathrm{L} / \mathrm{min}$, which approximates to a keratitis scenario. Although physiological tears are complex in nature, ${ }^{43}$ the electrolyte-only STF version used here represents a reasonable model in terms of mimicking and estimating the effect of hydrodynamic drug wash-out. Therefore, it can be hypothesised that the ability of contact lenses to deliver elevated amounts of drug is due, not only to the reservoir capacity within the lens matrix, but also to a protective effect conferred by the lens which acts to shield the underlying dose from wash out, especially at the lens-eye interface.

\subsection{Drug delivery to the eye - kinetic considerations}

Drug delivery to the cornea is a dynamic process, governed by a sequence of release and partitioning events. ${ }^{46}$ Firstly, drug must be released from its vehicle, involving dissociation from cyclodextrin or dissociation from cyclodextrin and the matrix of the hydrogel contact lens. Next, the drug must traverse the tear film followed by the mucus layer overlying the corneal epithelium. The drug must then penetrate the tight junctions that exist between cells of the epithelium, before finally entering the target tissue - the stroma. However, the stroma is not the diffusional sink as the drug can diffuse across the endothelium before entering the 
anterior chamber and deeper into the eye. Thus, when a drug dose applied to the cornea is absorbed, the concentration in the stroma will build up to a level depending on the driving force of the concentration gradient. Steady state may be obtained when the amount of drug entering the tissue is balanced by the amount exiting, i.e. entering the anterior chamber; otherwise the level reduces as the drug continues diffusing into the anterior chamber as indicated in Figure 3.

Table 1 shows that in the multiple dosing experiment, chlorhexidine reached steady state after 5 drops ( 6 hours) attaining a corneal dose of just over $1 \mu \mathrm{g}$ when dosed with $0.2 \%$. This was approximately $1 \%$ of the total applied dose, and the $\%$ delivered decreased thereafter. When dosed with $2 \%$, the chlorhexidine did not quite attain steady state within the maximum timeframe of the experiment (7 hours), although levels were clearly much higher than $0.2 \%$ by a factor of approximately x $10 ; \%$ delivered was approximately $0.75 \%$. Data obtained using either $0.2 \%$ and $2 \%$ highlights the large amount of chlorhexidine that fails to penetrate the cornea - this is clearly exacerbated by the STF irrigation, designed to simulate high levels of tear production seen in keratitis patients. This is investigated further below. When a single dose was applied, steady state cannot be attained. As the applied dose depletes, the driving force diminishes and the drug partitions into the anterior chamber, causing the resident drug in the stoma to decrease. Here, chlorhexidine resident in the cornea was determined at three timepoints $(2,6$ and $24 \mathrm{~h})$ following the application of single doses. Figure 3 shows chlorhexidine build up and depletion is apparent in most of the chlorhexidine delivery profiles. However, the level to which chlorhexidine built up, and subsequently diminished varied greatly depending on the manner in which it was applied, and whether or not there was constant irrigation with STF.

Vontobel et al (2015) demonstrated that chlorhexidine (or polyhexamethylene biguanide) did not readily permeate from $0.02 \%$ solution through the cornea to the anterior chamber through excised rabbit corneas, which may explain why treatment of Acanthamoeba keratitis requires many months of sustained topical drug administration. ${ }^{9}$ However, Figure 3 shows this not to be the case at the higher levels of 0.2 and $2 \%$.

\subsection{Effect of $\beta-\mathrm{CD}$ on delivery of chlorhexidine}

It is clear that delivery of chlorhexidine via 0.2 and $2 \%$ eyedrops was poor with no major accumulation in the cornea evident. Addition of $\beta-C D$ clearly enhanced drug delivery compared to eyedrops (by a factor of x 5 after 2 hours). However, although higher concentrations remained after $6 \mathrm{~h}$ compared to standard eyedrops, depletion of chlorhexidine 
from the cornea during STF irrigation still occurred. The efficacy of cyclodextrins in enhancing corneal drug penetration is typically attributed to an increase in solubilised concentration, thereby creating a higher concentration gradient and driving force. However, this logic does not apply to the current data in the same manner, as the chlorhexidine as digluconate salt is freely soluble in water.

The formation of 1:2 chlorhexidine: $\beta$-CD host complexes (Figure 6) was confirmed by the molecular modelling work as previously reported by Denadai et al (2007). ${ }^{38}$ As a digluconate salt, chlorhexidine is a symmetrical molecule consisting of a central hexamethylene bridge connecting two chlorophenyl and protonated biguanidine groups. As depicted in Figure 6c) and d) (circled in red), the chlorhexidine forms a stable complex with the two $\beta$-CD molecules, inserting the two hydrophobic terminal mono-chloro phenyl group inside the $\beta-C D$ cavities, whereas the central moiety of chlorhexidine bearing 2 x positive charges remains uncomplexed. However, the interactions are not particularly strong and the modelling simulation showed that the complex readily dissociates.

It is generally accepted that drug-cyclodextrin complexes cannot penetrate the corneal epithelium as a whole unit. However, there is evidence that cyclodextrins interact with the surface of the eye specifically by extracting cholesterol from the corneal epithelium, disrupting its integrity and rendering it more permeable. ${ }^{47}$ The sequestration of epithelium cholesterol from the cornea has been reported using a $30 \mathrm{mg} / \mathrm{mL} \beta-\mathrm{CD}$ solution, resulting in significant enhancement of the ocular delivery of riboflavin. ${ }^{19}$ This may explain why $\beta-C D$ enhanced delivery of chlorhexidine with the eyedrops, as the $\beta-C D$ molecules are free in solution and can act on the corneal epithelium. Using alternative cyclodextrins may produce better results: for example, the smaller $\alpha-C D$ which may be less sterically restrained. Conversely, cyclodextrin derivatives such as hydroxypropyl- $\beta-C D$ are likely to experience greater steric hindrance and lower levels of drug delivery enhancement.

\subsection{Effect of loaded contact lenses on delivery of chlorhexidine}

The effectiveness of contact lens delivery compared to eyedrops was clearly demonstrated in this work. There are several advantages of contact lenses over eyedrops in ocular drug delivery, including 1) protection from drug washout by tears 2) reduced frequency of dosing, with potential 0-order delivery 2) retention of high drug concentrations at the corneal surface, enabling the controlled and extended release of the drug, obviating nasolachrymal drainage (however, flux was not determined, as the cornea is not the ultimate sink and we were interested in the drug concentration resident within the cornea, hence potential activity 
against microbial infiltrates). The latter is clearly demonstrated in this work with an increase in corneal chlorhexidine between 2 and $6 \mathrm{~h}$ of contact lens wear, even with constant STF irrigation. In comparison, the amount of chlorhexidine delivered between these time intervals with eye drops decreased when irrigated with STF due to the washout effect on the applied eyedrops.

As expected, drug-loaded hydrogel contact lenses delivered a significantly greater amount of chlorhexidine to the cornea than eyedrops and this was reflected in the significant increase in the size of the zones of inhibition produced by treated, excised corneas. The contact lenses should not make physical contact with the cornea, rather, the two are separated by a thin tear film. ${ }^{48}$ As chlorhexidine is in the polar cationic charged state, the aqueous tear film presents no diffusional barrier between the lens and the corneal surface. This allows for increased ocular residence time of the drug and making the chlorhexidine far less susceptible to nasolacrimal drainage. At a concentration of $2.0 \%$, and to a lesser extent $0.2 \%$, chlorhexidine provided high levels of delivery from contact lens delivery. Chlorhexidine (as digluconate) bears two positive charges and binds to not only microbes, but also membrane components including surface mucous, however, by extracting the entire cornea it was not possible to differentiate between drug bound to the ocular surface and that that had penetrated deeper into the stroma.

In the current work, the lenses were loaded by immersion in drug solution. Although straightforward, the process is reported to have drawbacks such as low capacity and burst release. ${ }^{49}$ However, while the presented data show a prompt high concentration of chlorhexidine in the stroma - which is beneficial in providing rapid microbicidal action sustained release over the 24-hour study period was also observed, even with constant STF irrigation. Further controlled retardation of the release of chlorhexidine from contact lenses may be achieved by several methods such as altering the hydrophilicity of the lens monomers ${ }^{49}$ or the inclusion of other compounds, for example, vitamin $\mathrm{E}^{50}$ or hyaluronic acid. ${ }^{51}$ However, it would not be appropriate to seek to extend the use of contact lenses beyond the 24 hours used in this study, particularly given that improper/extended use of contact lenses may have been responsible for giving rise to the patient's condition in the first place. Replacing the drug-loaded contact lenses on a daily basis is regarded as good practice. ${ }^{52}$

As is typical in reservoir-type drug delivery devices (including transdermal patches), the dose delivered as a percentage of that loaded into the lens was low. For example, $\sim 4 \%$ of the loaded drug was delivered after $6 \mathrm{~h}$ of wear (with STF), highlighting that $2.0 \%$ chlorhexidine contact lenses are currently a rather inefficient delivery method as the majority 
of the drug is wasted, trapped inside the contact lens reservoir and not being released. ${ }^{53}$ However, the increased amount of drug delivered may make its use clinically relevant, perhaps in delivering a high concentration for a shorter time.

\subsection{Effect of $\beta-C D$ and loaded contact lenses on delivery of chlorhexidine}

Our hypothesis that chlorhexidine delivery to the cornea would be enhanced using a combination of both $\beta$-CD complexation and hydrogel contact lenses was not supported. Combinations of techniques have previously yielded success in ocular drug delivery, ${ }^{54}$ and $\beta$ $\mathrm{CD}$ has previously been shown to facilitate the controlled release of chlorhexidine from polymer matrices. ${ }^{29}$ However, no significant difference between the delivery of chlorhexidine from chlorhexidine-only loaded lenses and chlorhexidine- $\beta-C D$ lenses was found, as shown with the in vitro ocular delivery experiment. In fact, chlorhexidine- $\beta$-CD contact lenses delivered somewhat less than the chlorhexidine-only contact lenses. This was supported by the statistically similar zones of inhibition produced by contact lens-treated corneas (Figure 8).

From Figure 6 it was determined that the volume of the complex was $\sim 5 \mathrm{x}$ that of chlorhexidine. This would sterically restrict its ability to dissociate and liberate chlorhexidine within the hydrogel polymer matrix, and therefore release at the eye surface, as depicted in Figure 10. Consequently, there would be a reduced apparent concentration gradient than that achieved with chlorhexidine-only loaded lenses and thus less drug would be able to diffuse out of the contact lens and into the eye. This process could be coupled with less cholesterol sequestration by the free $\beta-\mathrm{CD}$ molecules. However, at 6 hours the $\beta-\mathrm{CD}$ chlorhexidine contact lens delivery results were similar to the chlorhexidine-only lenses, although after 24 hours, the amount of chlorhexidine delivered from $\beta$-CD containing lenses was significantly less than from those without $\beta-C D$, perhaps indicating the comparative steric trapping of complexed drug within the lens.

Whether or not the epithelium is ulcerated is significant regarding topical antibiotic penetration into the corneal stroma. Generally, topically applied compounds poorly penetrate the cornea when the epithelium is intact, although if ulceration has occurred the epithelium is evidently no longer a barrier. ${ }^{5}$ Indeed debridement of the corneal epithelium is practiced in some clinics to enhance the penetration of antimicrobials to the stroma.

\subsection{Potential clinical relevance - microbiology}


The correlation between chlorohexidine concentration in the cornea and zone of inhibition data (Figure 9) provided validation of the different capabilities of the various drug delivery approaches evaluated.

The reported $\mathrm{MIC}_{90}$ of chlorhexidine for Aspergillus spp. and Fusarium spp., the most common fungi associated with fungal keratitis, are $8.6-16 \mu \mathrm{g} / \mathrm{mL}$ and $2-8 \mu \mathrm{g} / \mathrm{mL}$, respectively, ${ }^{55}$ while the MIC for Acanthamoeba is approximately $6.25 \mu \mathrm{g} / \mathrm{mL} .{ }^{56} \mathrm{We}$ can estimate whether sufficient chlorhexidine to inhibit microbial growth has been delivered to the cornea by using the corneal volume to deduce the local concentration. The volume of a standard porcine cornea has been previously determined as $0.190 \mathrm{~mL} /$ cornea $^{57}$. This value was used in the equation below to determine the local chlorhexidine concentration values:

$$
\text { Conc }(\mu g / m L)=\frac{\mu g \text { per cornea }}{\text { Volume }_{\text {cornea }}}
$$

Based on this, delivery by singly dosed eyedrops was unsurprisingly poor and chlorhexidine levels would not attain the MICs against any of the above microorganisms beyond the $2 \mathrm{~h}$ time point, suggesting that they would need to be dosed frequently in order to achieve consistent therapeutic concentrations in the cornea. Based on the values in Figure 3, with STF irrigation eyedrops without $\beta$-CD produced a local chlorhexidine concentration of $9.5 \mu \mathrm{g} / \mathrm{mL}$ $(1.8 \mu \mathrm{g} /$ cornea) $2 \mathrm{~h}$ after exposure but this reduced to $2.3 \mu \mathrm{g} / \mathrm{mL}(0.43 \mu \mathrm{g} /$ cornea $)$ at $6 \mathrm{~h}$. Chlorhexidine eyedrops containing $\beta-\mathrm{CD}$ showed an improvement over those without, delivering and maintaining chlorhexidine concentrations of $61.6 \mu \mathrm{g} / \mathrm{mL}(11.71 \mu \mathrm{g} / \mathrm{cornea})$, $42.8 \mu \mathrm{g} / \mathrm{mL}(8.13 \mu \mathrm{g} / \mathrm{cornea})$ and $27.0 \mu \mathrm{g} / \mathrm{mL}(5.13 \mu \mathrm{g} /$ cornea $)$ at 2, 6 and $24 \mathrm{~h}$, respectively, indicating their superiority to chlorhexidine-only eyedrops and suggesting that such eyedrops may not need to be dosed as frequently in order to maintain therapeutic concentrations in vivo. However, chlorhexidine loaded contact lenses delivered drug concentrations far surpassing the MICs for Aspergillus spp., Fusarium spp. and Acanthamoeba, both with and without $\beta-\mathrm{CD}$, demonstrating the superiority of contact lenses for delivering chlorhexidine to a clinically beneficial level compared to delivery from eyedrops. Even after $24 \mathrm{~h}$ with the poorer performing $\beta$-CD containing contact lens, $53.7 \mu \mathrm{g} / \mathrm{mL}(10.21 \mu \mathrm{g} /$ cornea $)$ chlorhexidine was present in the corneas - over 3 times the MIC for the least susceptible microorganism considered.

4.5 Potential clinical relevance - cytotoxicity 
Chlorhexidine is known to be cytotoxic; however, it continues to be widely used in, for example, personal care products for the buccal cavity. Typically, $0.02 \%-0.2 \%$ chlorhexidine solutions are used in ocular therapeutics. ${ }^{58}$ Vontobel et al (2015) reported no toxicity to or disruption of the barrier function of the corneal epithelium from a $0.02 \%$ solution. ${ }^{9}$ Ocampo (2005) reported that no severe inflammation and structural alterations in the deep layers of the cornea in rabbit eyes when dosed with multiple applications of $0.2 \%$ chlorhexidine gluconate as a pure solution; although this contradicted an earlier report where toxicity was noted. ${ }^{59,60}$ Anecdotal reports from field trials indicate that the use of $0.2 \%$ chlorhexidine drops for treating fungal keratitis is tolerated, with a few incidences of initial stinging and mild surface toxicity - which resolves and is much milder than that seen with some other eye drops, such as fortified gentamicin - also used in severe ocular infections (Burton, personal communication). Tabor et al, (1989) and Bever et al (2016) both reported that $4 \%$ solution were toxic and caused opacification of the cornea. ${ }^{61,62}$ Intravitreal injection of aqueous $0.1 \%$ chlorhexidine was associated with a low rate of post-injection endophthalmitis and was well tolerated by patients. ${ }^{63}$ Together these reports indicate a concentration dependence to ocular toxicity although they do not provide information as to the levels of chlorhexidine delivered to the cornea, which will be formulation-dependent. Interestingly, Teixeira et al (2015) reported that $\alpha-C D, \beta-C D$ and HP- $\beta-C D$ increased the antimicrobial activity of chlorhexidine, but decreased its cytotoxic effects on mammalian fibroblasts and osteoblasts. ${ }^{64}$

Some Acanthamoeba and fungal keratitis infections are considered untreatable with current drug therapies, including $0.02 \%$ to $0.06 \%$ chlorhexidine eyedrops. Therefore, higher concentrations of chlorhexidine may be justified since the risk of vision loss in these cases is very high. ${ }^{65,66}$ A carefully monitored dose from a lens containing $2 \%$ chlorhexidine could be justifiable, where the greater drug penetration would allow it to target deeply embedded infiltrates. However, this would need to be considered against the elevated risk of toxicity. ${ }^{66}$

\section{Conclusion}

Addition of $\beta-C D$ to chlorhexidine eyedrops enhanced drug delivery to the cornea relative to control; however, drug loaded contact lenses alone proved much superior and delivered concentrations surpassing the MIC against causative fungi involved in keratitis throughout the 24-hour study period. The $\beta$-CD eyedrops may have application in corneal ulceration, whereas the loaded lens would be more applicable for treating non-ulcerated infiltrates. From 
a methodological standpoint, the use of appropriate STF irrigation should be practiced in future in vitro ocular delivery experiments to ensure delivery rates are not over-estimated.

\section{Acknowledgement}

The authors gratefully acknowledge the support of the BBSRC via a Follow on Fund (BB/N022106/1) and Super Follow on Fund (BB/S004874/1), and Life Science Research Network Wales, an initiative funded through the Welsh Government Sêr Cymru program.

\section{Declaration of interest statement}

The authors report no conflict of interest.

\section{References}

1. Wu, J., Zhang, W., Zhao, J. and Zhou, H. Review of clinical and basic approaches of fungal keratitis. International Journal of Ophthalmology 2016, 9, 1676-1683.

2. Chowdhary, A., Singh, K. Spectrum of fungal keratitis in North India. Cornea, 2005, 24, 8-15.

3. Brooks, D.E. Equine keratomycosis: An international problem. Equine Veterinary Education 2009, 21, 243-246. doi: 10.2746/095777309X409929

4. Knop, E., Knop, N. The role of eye-associated lymphoid tissue in corneal immune protection. Journal of Anatomy 2005, 206, 271-285.

5. Dos Santos, D.L., Kwitko, S., Marinho, D.R., de Araújo, B.S., Locatelli, C.I., Rott, M.B. Acanthamoeba keratitis in Porto Alegre (southern Brazil): 28 cases and risk factors. Parasitology Research 2018, 117, 747-750.

6. Kent, C. Winning the battle against corneal ulcers. Review of Ophthalmology. September 2013.

7. Ansari, Z., Miller, D., Galor, A. Current thoughts in fungal keratitis: diagnosis and treatment. Current Fungal Infection Reports 2013, 7, 209-218. 
8. Heredero-Bermejo, I., Sánchez-Nieves, J., Soliveri, J., Gómez, R., de la Mata, F., CopaPatiño, J., Pérez-Serrano, J. In vitro anti-Acanthamoeba synergistic effect of chlorhexidine and cationic carbosilane dendrimers against both trophozoite and cyst forms. International Journal of Pharmaceutics 2016, 509, 1-7.

9. Vontobel, S.F., Abad-Villar, E.M., Kaufmann, C., Zinkernagel, A.S., Hauser, P.C., Thiel, M.A. Corneal penetration of polyhexamethylene biguanide and chlorhexidine digluconate. Journal of Clinical \& Experimental Ophthalmology, 2015, 6:430. doi:10.4172/21559570.1000430.

10. Pratten, J., Barnett, P., Wilson, M. Composition and susceptibility to chlorhexidine of multispecies biofilms of oral bacteria. Applied Environmental Microbiology 1998 64, 35153519.

11. Fathilah, A.R., Himratul-Aznita, W.H., Fatheen, A.R., Suriani, K.R. The antifungal properties of chlorhexidine digluconate and cetylpyrinidinium chloride on oral Candida. Journal of Dentistry 2012, 40, 609-615.

12. Park, J.B., Park, N.H. Effect of chlorhexidine on the in vitro and in vivo herpes simplex virus infection. Oral Surgery, Oral Medicine, Oral Pathology, and Oral Radiology 1989, 67, 149-153.

13. Rahman, M., Johnson, G., Husain, R., Howlader, S., Minassian, D. Randomised trial of $0.2 \%$ chlorhexidine gluconate and $2.5 \%$ natamycin for fungal keratitis in Bangladesh. British Journal of Ophthalmology 1998, 82, 919-925.

14. Kosrirukvongs, P., Wanachiwanawin, D., Visvesvara, G.S. Treatment of Acanthamoeba keratitis with chlorhexidine. Ophthalmology 1999, 106, 798-802.

15. Martin, M., Rahman, M., Johnson, G., Srinivasan, M., Clayton, Y. Mycotic keratitis: Susceptibility to antiseptic agents. International Ophthalmology 1995, 19, 299-302.

16. Lim, N., Goh, D., Bunce, C., Xing, W., Fraenkel, G., Poole, T., Ficker, L. Comparison of polyhexamethylene biguanide and chlorhexidine as monotherapy agents in the treatment of acanthamoeba keratitis. American Journal of Ophthalmology 2008, 145, 130-135. 
17. Schwach-Adbellaoui, K., Vivien-Castioni, N., Gurny, R. Local delivery of antimicrobial agents for the treatment of periodontal diseases. European Journal of Pharmaceutics and Biopharmaceutics 2000, 50, 83-99.

18. Leikin, J.B., Paloucek, F.P. Chlorhexidine gluconate. In Poisoning and Toxicology Handbook $4^{\text {th }}$ Edition. CRC Press. Boca Raton, USA, 2008; pp 183-184.

19. Morrison, P.W.J., Khutoryanskiy, V.V. Anatomy of the eye and the role of ocular mucosa in drug delivery. In Khutoryanskiy, V.V. ed. Mucoadhesive Materials and Drug Delivery Systems. Wiley, New Jersey, 2014, pp 39-60.

20. Prajna, N. Comparison of natamycin and voriconazole for the treatment of fungal keratitis. Archives of Ophthalmology 2010, 128, 672.

21. O'Day, D.M., Ray, W.A., Head, S.W., Robinson, R.D. Influence of the corneal epithelium on the efficacy of topical antifungal agents. Investigative Ophthalmology \& Visual Science 1984, 25, 855-859.

22. Li, C., Chauhan, A. Ocular transport model for ophthalmic delivery of timolol through pHEMA contact lenses. Journal of Drug Delivery Science and Technology 2007, 17, 69-79.

23. Phan, C., Subbaraman, L., Jones, L. Contact lenses for antifungal ocular drug delivery: a review. Expert Opinion on Drug Delivery 2014, 11, 537-546.

24. Djebli, N., Khier, S., Griguer, F., Coutant, A., Tavernier, A., Fabre, G., Leriche, C., Fabre, D. Ocular drug distribution after topical administration: population pharmacokinetic model in rabbits. European Journal of Drug Metabolism and Pharmacokinetics 2016, 42, 5968.

25. Washington, N., Washington, C., Wilson, C.G. Ocular Drug Delivery. In, Physiological Pharmaceutics: Barriers to Drug Absorption. 2nd ed., CRC Press, Boca Raton, USA, 2001 pp 249-269.

26. Jain, M.R. Drug delivery through soft contact lenses. British Journal of Ophthalmology 1988, 72, 150-154.

27. Pathak, Y., Sutariya, V., Hirani, A. Nano-biomaterials for ophthalmic drug delivery. Switzerland: Springer, 2016. 
28. Cortés, M., Sinisterra, R., Avila-Campos, M., Tortamano, N., Rodney Garcia, R. The Chlorhexidine: beta-cyclodextrin inclusion compound: preparation, characterization and microbiological evaluation. Journal of Inclusion Phenomena and Macrocyclic Chemistry 2001, 40, 297-302.

29. Lavoine, N., Tabary, N., Desloges, I., Martel, B., Bras, J. Controlled release of chlorhexidine digluconate using $\beta$-cyclodextrin and microfibrillated cellulose. Colloids and Surfaces B: Biointerfaces 2014, 121, 196-205.

30. Kettel, M., Heine, E., Schaefer, K., Moeller, M. Chlorhexidine loaded cyclodextrin containing PMMA nanogels as antimicrobial coating and delivery systems. Macromolecular Bioscience 2016, 17 ID201600230. Available at:

https://onlinelibrary.wiley.com/doi/abs/10.1002/mabi.201600230

31. Kumar, A., Jha, G. Drug delivery through soft contact lenses: An introduction. Chronicles of Young Scientists 2011 2, 3.

32. Maulvi, F., Soni, T.G., Shah, D.O. A review on therapeutic contact lenses for ocular drug delivery. Drug Delivery 2016, 23, 3017-3026.

33. Stjernschantz, J, Astin, M. Anatomy and physiology of the eye. Physiological aspects of ocular drug therapy. In Biopharmaceutics of Ocular Drug Delivery, Edman, P. Ed, CRC Press, Boca Raton, USA.

34. Schoenwald, R. Ocular drug delivery: Pharmacokinetic considerations. Clinical Pharmacokinetics 1990, 18, 255-269.

35. Denadai, A.M.L., Santoro, M.M., Da Silva, L.H., Viana, A.T., Santos, R.A.S., Sinisterra, R.D. Self-assembly characterization of the $\beta$-cyclodextrin and hydrochlorothiazide system: NMR, phase solubility, ITC and QELS. Journal of Inclusion Phenomena and Macrocyclic Chemistry 2006, 55, 41-49.

36. Teweldemedhin, M., Gebreyesus, H., Atsbaha, A.T., Asgedom, S.W., Saravanan, M. Bacterial profile of ocular infections: a systematic review. BMC Ophthalmology 2017, 17, Article number 212.

37. Qi, H., Nishihata, T., Rytting, J.H. Study of the interaction between $\beta$-cyclodextrin and chlorhexidine. Pharmaceutical Research 1994, 11, 1207-1210. 
38. Denadai, Â., Teixeira, K., Santoro, M., Pimenta, A., Cortés, M., Sinisterra, R.

Supramolecular self-assembly of $\beta$-cyclodextrin: an effective carrier of the antimicrobial agent chlorhexidine. Carbohydrate Research 2007, 342, 2286-2296.

39. Higuchi, T., Connors, K. A. Phase solubility techniques. Adv Anal Chem Instr 1965, $4,117-212$.

40. Samuelsen, L., Holm, R., Lathuile, A., Schönbeck, C. Correlation between the stability constant and $\mathrm{pH}$ for $\beta$-cyclodextrin complexes. Int J Pharm 2019, 568, 118523.

41. Shah, J., Vasanti, S., Anroop, B., Vyas, H. Enhancement of dissolution rate of valdecoxib by solid dispersions technique with PVP K 30 \& PEG 4000, preparation and in vitro evaluation. J Inclusion Phenom Macrocyclic Chem 2009, 63, 219-224.

42. Stickler, D.J., Thomas, B., Chawla, J.C. Antiseptic and antibiotic resistance in Gramnegative bacteria causing urinary tract infection in spinal cord injury patients. Paraplegia, 1981, 19, 50-58.

43. Achi, D., Alhanout, K., Piccerelle, P., Andrieu, V. Recent advances in ocular drug delivery. Drug Development and Industrial Pharmacy 2012, 39, 1599-1617.

44. De Koning, E.W., van Bijsterveld O.P. Schirmer test values and lysozyme content of tears in acute dendritic keratitis. Investigative Ophthalmology \& Visual Science 1984, 25, 5558.

45. Lal, H., Ahluwalia, B.K., Khurana, A.K., Sharma, S.K., Gupta, S. Tear lysozyme levels in bacterial, fungal and viral corneal ulcers. Acta Ophthalmologica 1991, 69, 530-532.

46. Bachu, R. D., Chowdhury, P., Al-Saedi, Z., Karla, P. K., Boddu, S. Ocular drug delivery barriers-role of nanocarriers in the treatment of anterior segment ocular diseases.

Pharmaceutics 2018, 10, 28. doi:10.3390/pharmaceutics10010028

47. López, C., de Vries, A., Marrink, S. Molecular mechanism of cyclodextrin mediated cholesterol extraction. PLoS Computational Biology 2011, 7, p.e1002020.

48. Guzman-Aranguez, A., Colligris, B., Pintor, J.. Contact lenses: promising devices for ocular drug delivery. Journal of Ocular Pharmacology and Therapeutics 2013, 29, 189-199. 
49. Hu, X., Hao, L., Wang, H., Yang, X., Zhang, G., Wang, G., Zhang, X. Hydrogel contact lens for extended delivery of ophthalmic drugs. International Journal of Polymer Science, 2011, ID 814163. Available at: http://dx.doi.org/10.1155/2011/814163.

50. Paradiso, P., Serro, A., Saramago, B., Colaço, R., Chauhan, A. Controlled release of antibiotics from vitamin e-loaded silicone-hydrogel contact lenses. Journal of Pharmaceutical Sciences 2016, 105, 1164-1172.

51. Guidi, G., Korogiannaki, M., Sheardown, H. Modification of timolol release from silicone hydrogel model contact lens materials using hyaluronic acid. Eye \& Contact Lens: Science \& Clinical Practice 2014, 40, 269-276.

52. Kaplan, E. Extended wear: still an option? Review of Cornea and Contact Lenses 2017 November/December.

53. Hsu, K., Gause, S., Chauhan, A. Review of ophthalmic drug delivery by contact lenses. Journal of Drug Delivery Science and Technology 2014, 24, 123-135.

54. Rupenthal, I.D. Drug-device combination approaches for delivery to the eye. Current Opinion in Pharmacology 2017, 36, 44-51.

55. Sandle, T., Vijayakumar, R., Saleh Al Aboody, M., Saravanakumar, S. In vitro fungicidal activity of biocides against pharmaceutical environmental fungal isolates. Journal of Applied Microbiology 2014, 117, 1267-1273.

56. Syam, P. Persistent acanthamoeba keratitis in a non-contact lens wearer following exposure to bird seed dust. British Journal of Ophthalmology 2005, 89, 388-389.

57. Morgan, S. R., Pilia, N., Hewitt, M., Moses, R. L., Moseley, R., Lewis, P. N., Morrison, P. W. J., Kelly, S. L., Parker, J. E., Whitaker, D., Quantock, A. J., Heard, C. M. Controlled in vitro delivery of voriconazole and diclofenac to the cornea using contact lenses for the treatment of acanthamoeba keratitis. International Journal of Pharmaceutics, in press.

58. Geffen, N., Norman, G., Kheradiya, N., Assia, E. Chlorhexidine gluconate $0.02 \%$ as adjunct to primary treatment for corneal bacterial ulcers. Israel Medical Association Journal 2009, 11, 664-668. 
59. Ocampo Jr V.V., Yu E., Zhao, T.Z., Feroze, A.H., Foster, S. Toxicity of $0.2 \%$ chlorhexidine gluconate on the cornea and adjacent structures. Philippines Journal of Ophthalmology 2005, 30, 119-123.

60. Hamill, M., Osato, M., Wilhelmus, K. Experimental evaluation of chlorhexidine gluconate for ocular antisepsis. Antimicrobial Agents and Chemotherapy 1984, 26, 793-796.

61. Tabor, E., Bostwick, D.C., Evans, C. Corneal damage due to eye contact with chlorhexidine gluconate. Journal of the American Medical Association 1989, 261, 557-558. 62. Bever, G.J., Brodie, F.L., Hwang, D.G. Corneal injury from presurgical chlorhexidine skin preparation. World Neurosurgery 2016, 96, 610.

63. Merani, R., McPherson, Z.E., Luckie, A.P., Gilhotra, J.S., Runciman, J., Durkin, S., Muecke, J, Donaldson, M., Aralar, A., Rao, A., Davies, P.E. Aqueous chlorhexidine for intravitreal injection antisepsis. Ophthalmology 2016, 123, 2588 - 2594.

64. Teixeira, K.I., Denadai, A.M., Sinisterra, R.D., Cortés, M.E. Cyclodextrin modulates the cytotoxic effects of chlorhexidine on microrganisms and cells in vitro. Drug Delivery 2015, $22,444-453$.

65. Dart, J.K., Saw, V.P., Kilvington, S. Acanthamoeba keratitis: diagnosis and treatment update 2009. American Journal of Ophthalmology 2009, 148, 487-499.e2. doi: 10.1016/j.ajo.2009.06.009. Epub 2009 Aug 5.

66. Mathers, W. Use of higher medication concentrations in the treatment of Acanthamoeba keratitis. JAMA Ophthalmology 2006, 124, 923. doi:10.1001/archopht.124.6.923 


\section{Legends to figures}

Figure 1. Chemical structures of $\beta$-cyclodextrin and chlorhexidine.

Figure 2. Schematic representation of drug-loaded contact lens in situ, showing a higher concentration gradient providing increased drug delivery to the stroma, as described by Fick's laws of diffusion.

Figure 3. Time course profiles for the delivery of chlorhexidine into porcine corneas over 24 $\mathrm{h}(\mathrm{n}=3 \pm \mathrm{SD})$. Key and rank order (highest to lowest at $6 \mathrm{~h})$ : CL (contact lens) $>$ BCD+CL ( $\beta$ cyclodextrin + contact lens) $>\mathrm{CL}+\mathrm{STF}$ (contact lens + simulated tear fluid irrigation) $>\mathrm{CD}$ $(\beta$-cyclodextrin $)>\mathrm{CD}+\mathrm{STF}(\beta$-cyclodextrin + simulated tear fluid irrigation $)>\mathrm{D}$ (eyedrop alone) $>$ D+STF (eyedrop + simulated tear fluid irrigation).

Figure 4. ${ }^{1} \mathrm{H}$ NMR spectra. a) chlorhexidine, b) chlorhexidine- $\beta-C D$, c) superimposition of the two spectra, $\left(500 \mathrm{MHz}\right.$, in $\mathrm{H}_{2} \mathrm{O}$ ) and d) a representation of chlorhexidine with both phenyl groups located with two $\beta$-CD molecules.

Figure 5. Space-filling representations of chlorhexidine (above) and chlorhexidine inclusion complex involving two $\beta$-CD molecules.

Figure 6. Chlorhexidine- $\beta$-CD molecular dynamic simulations. The graph shows the variations of the van der Waals energy related to chlorhexidine during the simulation. The four images are the frames representative of the different states of the system during the simulation. The images a) and b) (circled in blue), show the position and orientation of chlorhexidine in the system when the van der Waals energy is the highest. In this condition, the chlorhexidine molecules are dispersed in the water system and distant from $\beta-C D$ complex. In the images c) and d) (circled in red), the chlorhexidine forms a complex with the two $\beta$-CDs, inserting the two mono-chloro phenyl group inside the $\beta$-CD cavities. In this conformation the system energy is the lowest, and consequently the most stable condition.

Figure 7. Plot showing the variations of binding free energy $(\Delta G)$ of $\beta-C D$ - chlorhexidine inclusion complexation during the simulation. The energy data were collected every 10000 picoseconds (ps) starting from 250000 ps. The graph clearly shows the ligand binding and 
releasing mechanism of $\beta-C D$ - chlorhexidine complex, with a wide variety of binding free energy during the desolvation process $(\Delta \mathrm{G} \max =1.24)$ and binding process $(\Delta \mathrm{G} \min =-$ 87.67).

Figure 8. Zone of inhibition analysis for chlorhexidine released from treated corneas against E. coli. In accordance with the amounts of chlorhexidine quantified by HPLC, the zones of inhibition produced by corneas treated with contact lenses containing only chlorhexidine exhibited the largest zone of inhibition $(30.3 \pm 1.0 \mathrm{~mm})$ of all conditions tested (Figure $7 \mathrm{a}$ ), whilst chlorhexidine eyedrops delivered the least active drug to corneas, producing the smallest observed zone of inhibition (Figure $7 \mathrm{~b}$ ) of $18.3 \pm 2.9 \mathrm{~mm}$ (note: measurements included the diameter of the well which was $15 \mathrm{~mm}$ ). When treated using chlorhexidine- $\beta$ CD contact lenses, wells containing the excised cornea produced a zone of inhibition of 27.8 $\pm 0.8 \mathrm{~mm}$ compared to $30.3 \pm 1.0 \mathrm{~mm}$ with the chlorhexidine contact lenses, as seen in Figure $8 \mathrm{c}$.

Figure 9. Correlation of zone of inhibition versus chlorhexidine delivery to the cornea for the different formulations tested. Inhibition zone diameters were converted to area $\left(\mathrm{mm}^{2}=\pi \mathrm{x}\right.$ $\left.(\mathrm{mm} / 2)^{2}\right)$ and corrected for the area of the $15 \mathrm{~mm}$ diameter well (i.e. inhibition zone area well area). Corrected inhibition zone area $=22.56 \times$ [CHX delivered].

Figure 10. Schematic representation of cyclodextrin-chlorhexidine inclusion complexes loaded into contact lens. Dissociation, or drug release is sterically inhibited at point X due to proximity to polymeric matrix. This assists rationalising the unexpectedly reduced corneal delivery of chlorhexidine observed when using chlorhexidine- $\beta-C D$ contact lenses. 
Fig 1
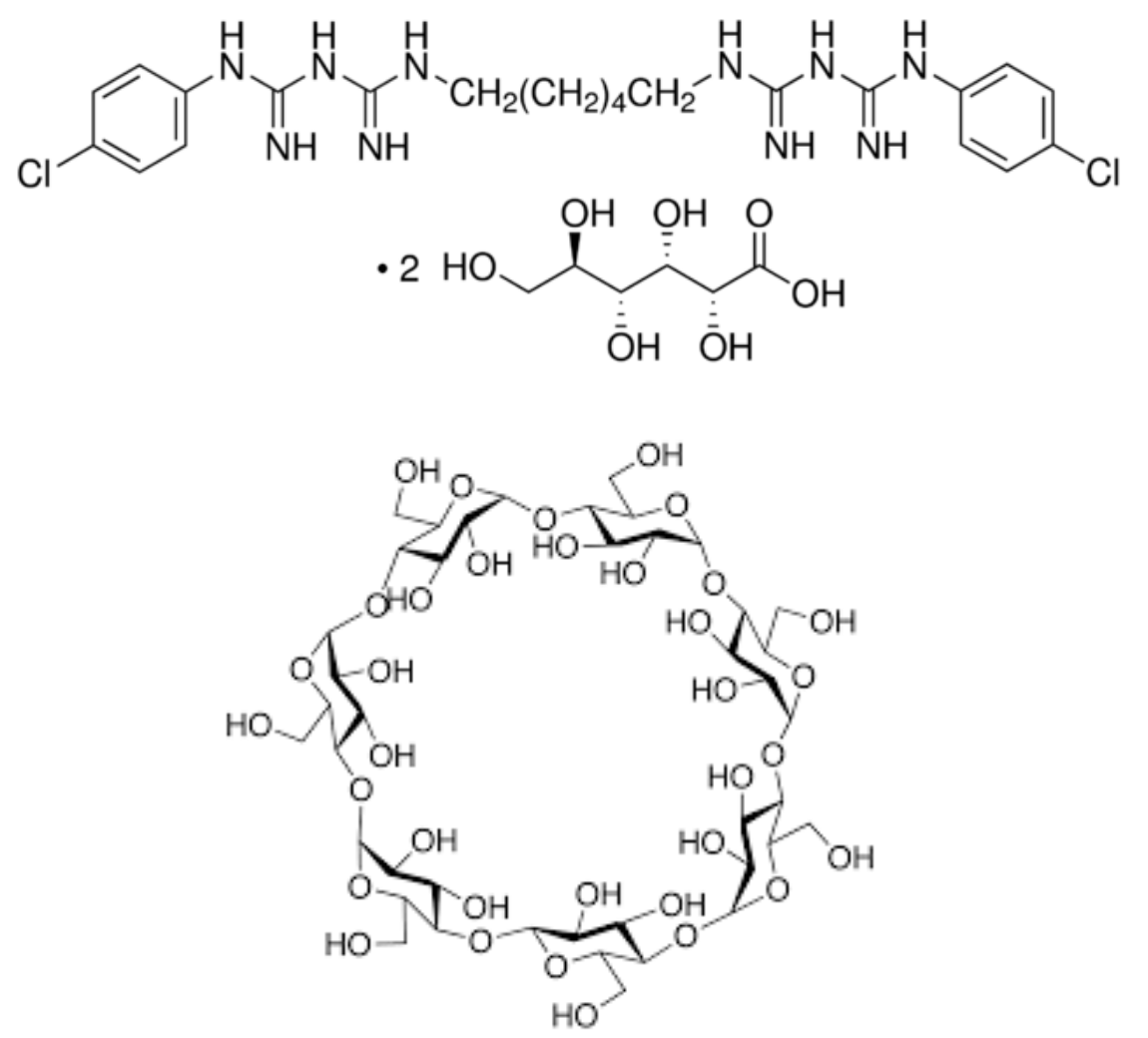
Fig 2

low concentration gradient

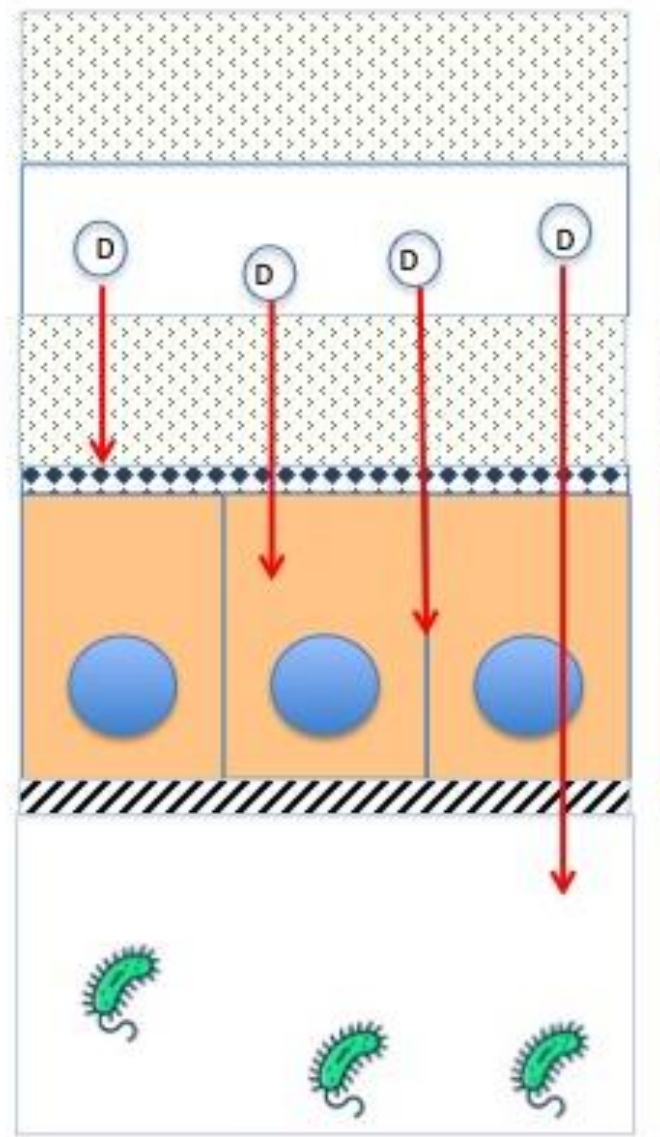

high concentration gradient
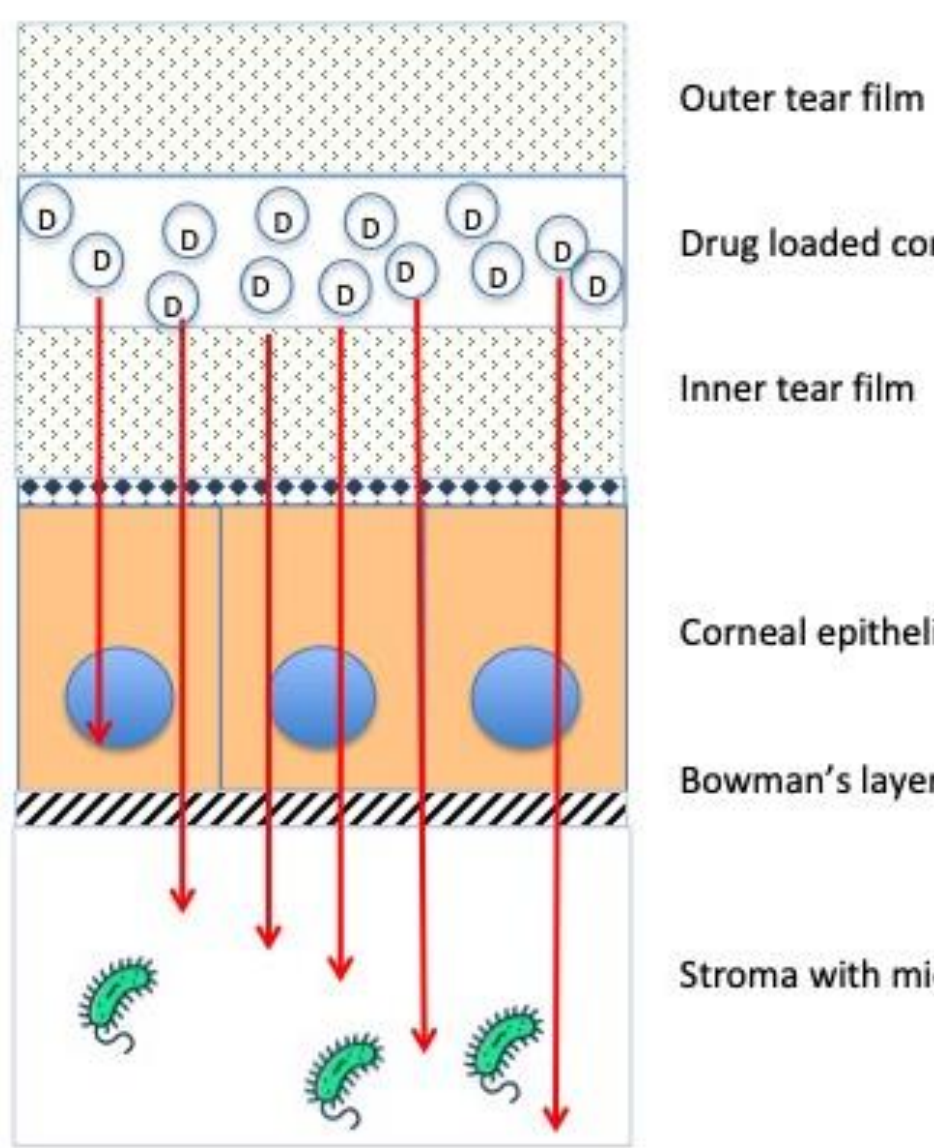

Drug loaded contact lens

Inner tear film

Corneal epithelium

Bowman's layer

Stroma with microbial load 


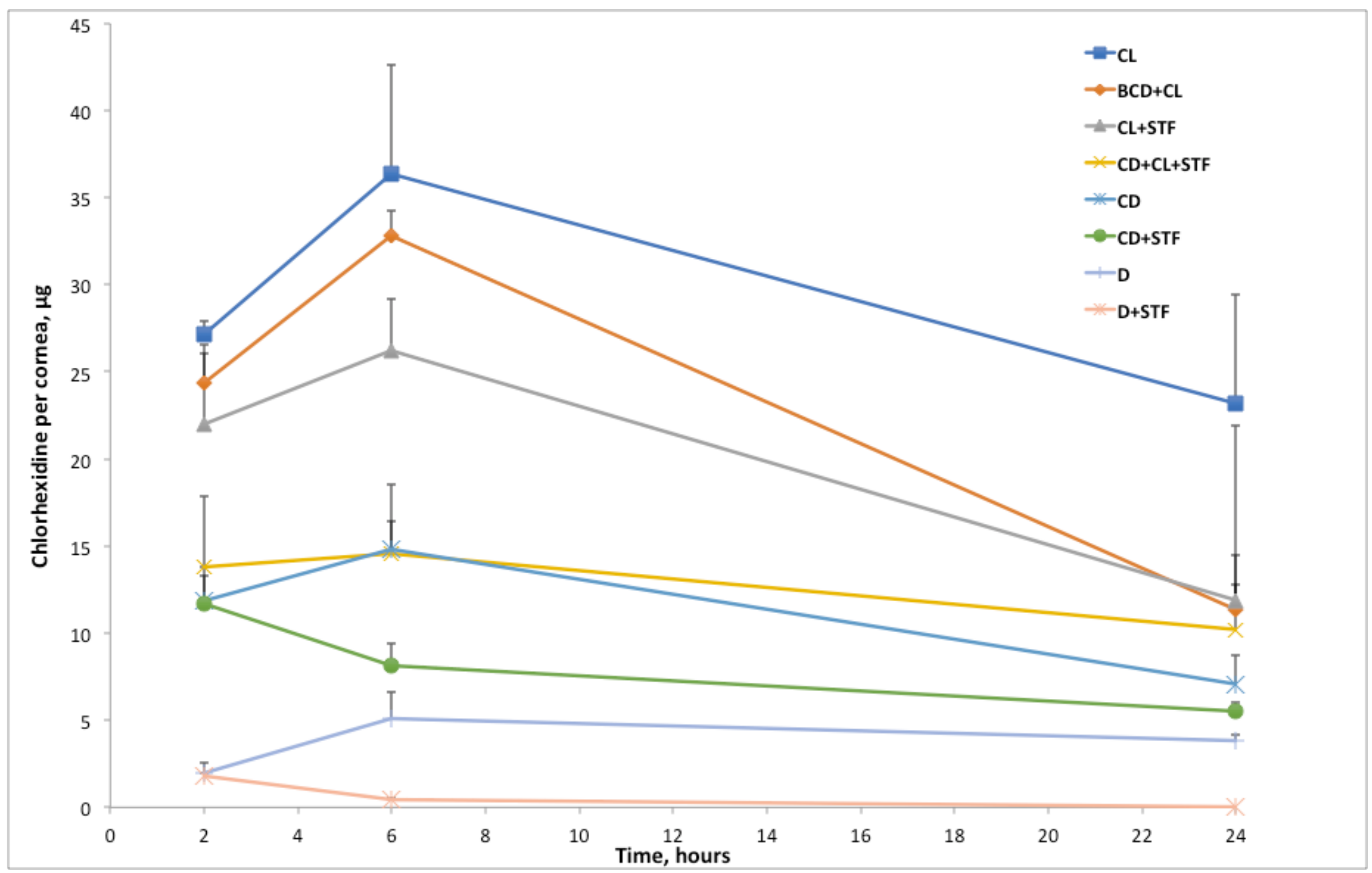



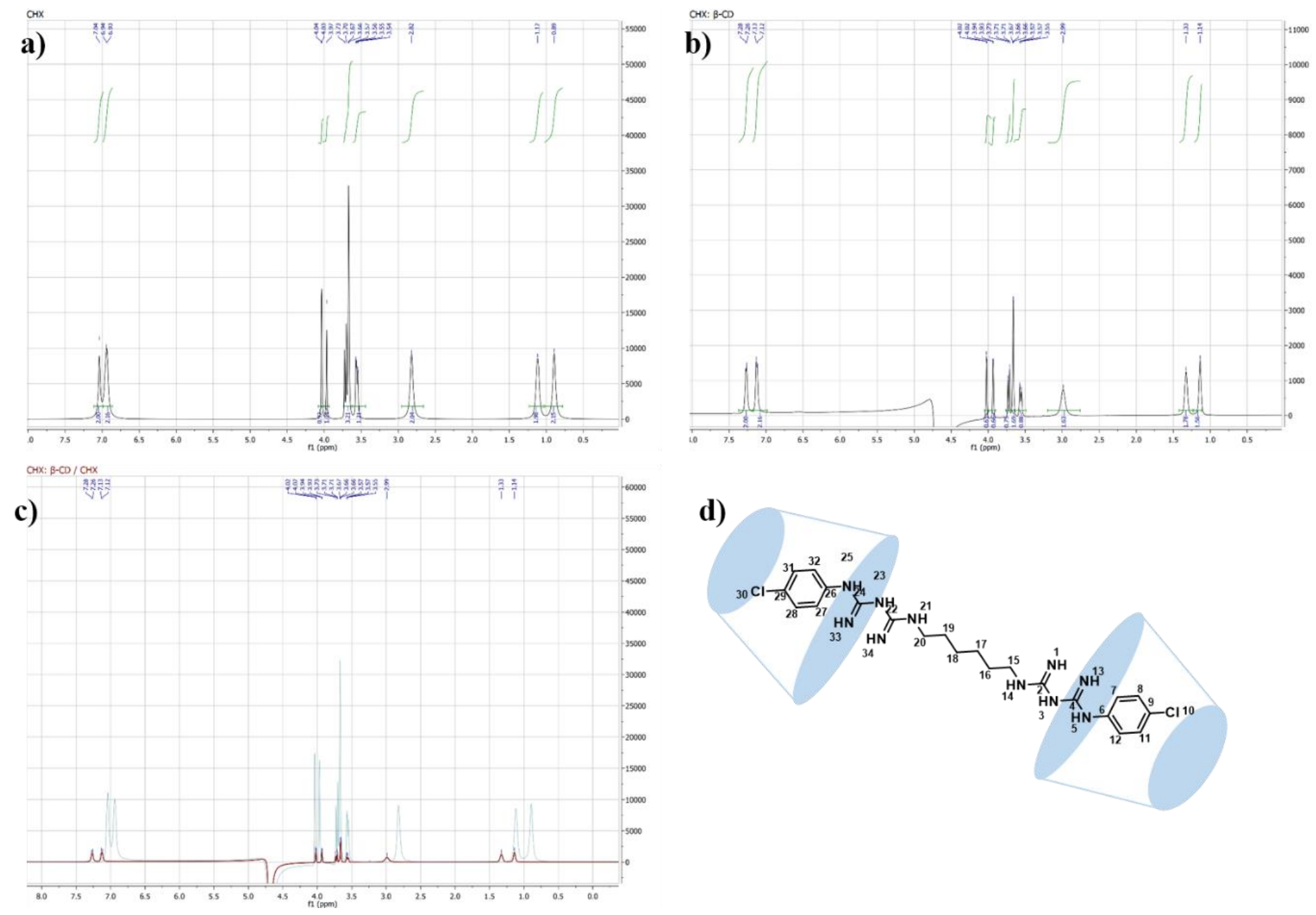

d)

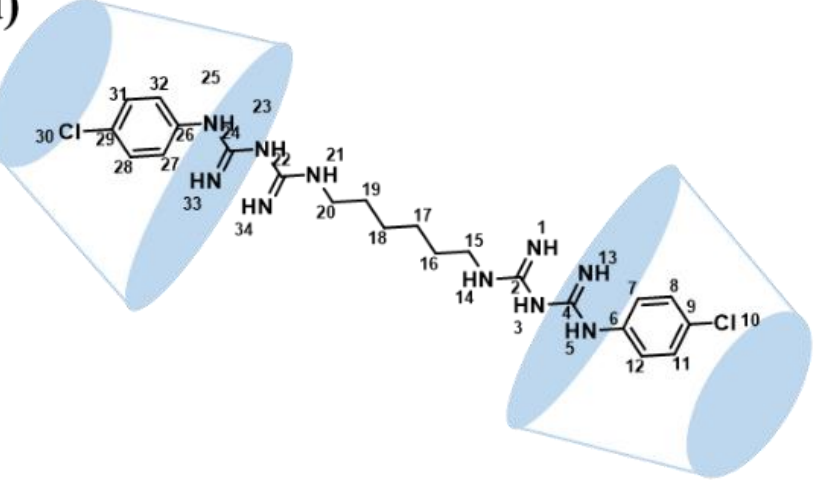


Fig 5

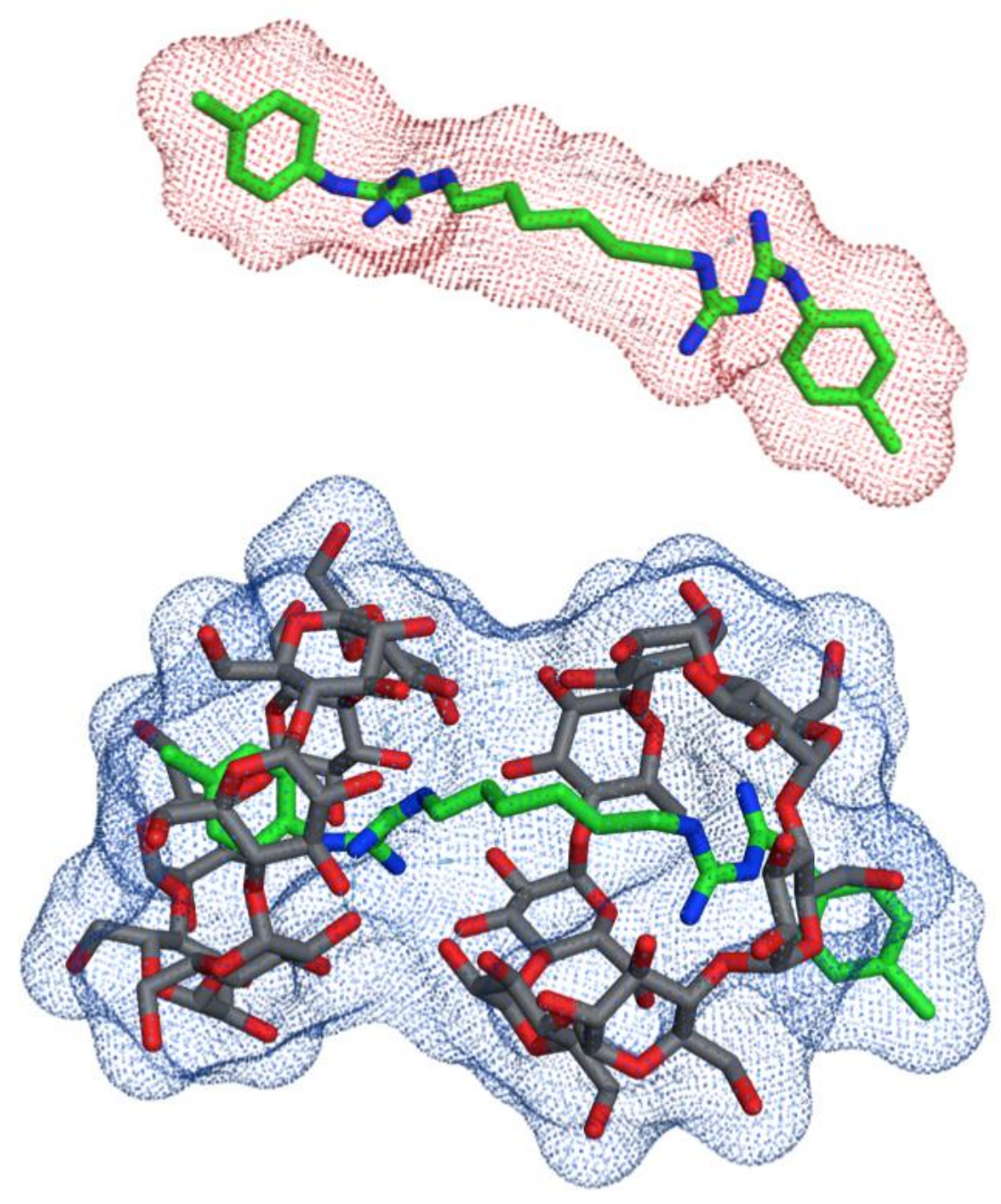




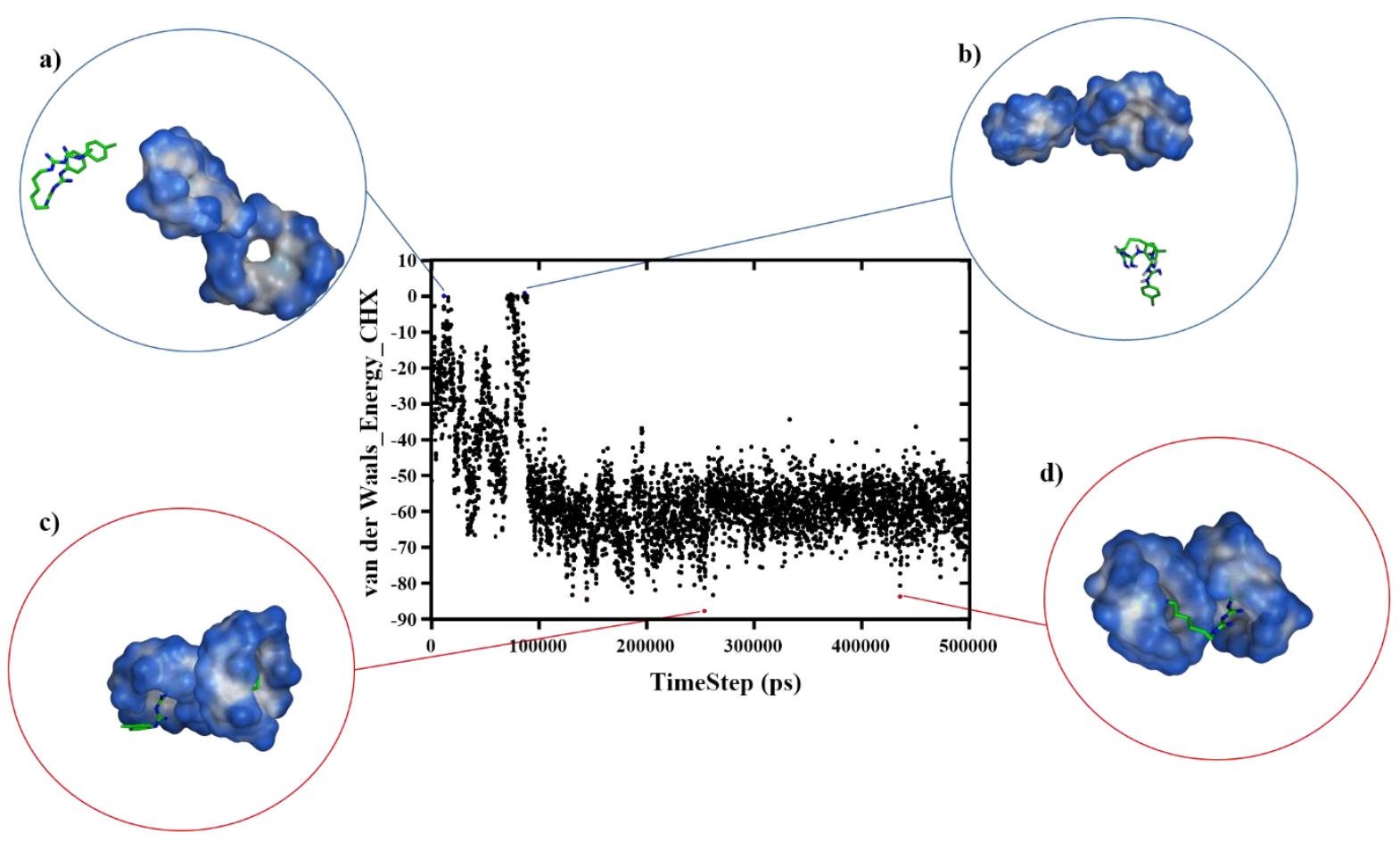


Figure 7

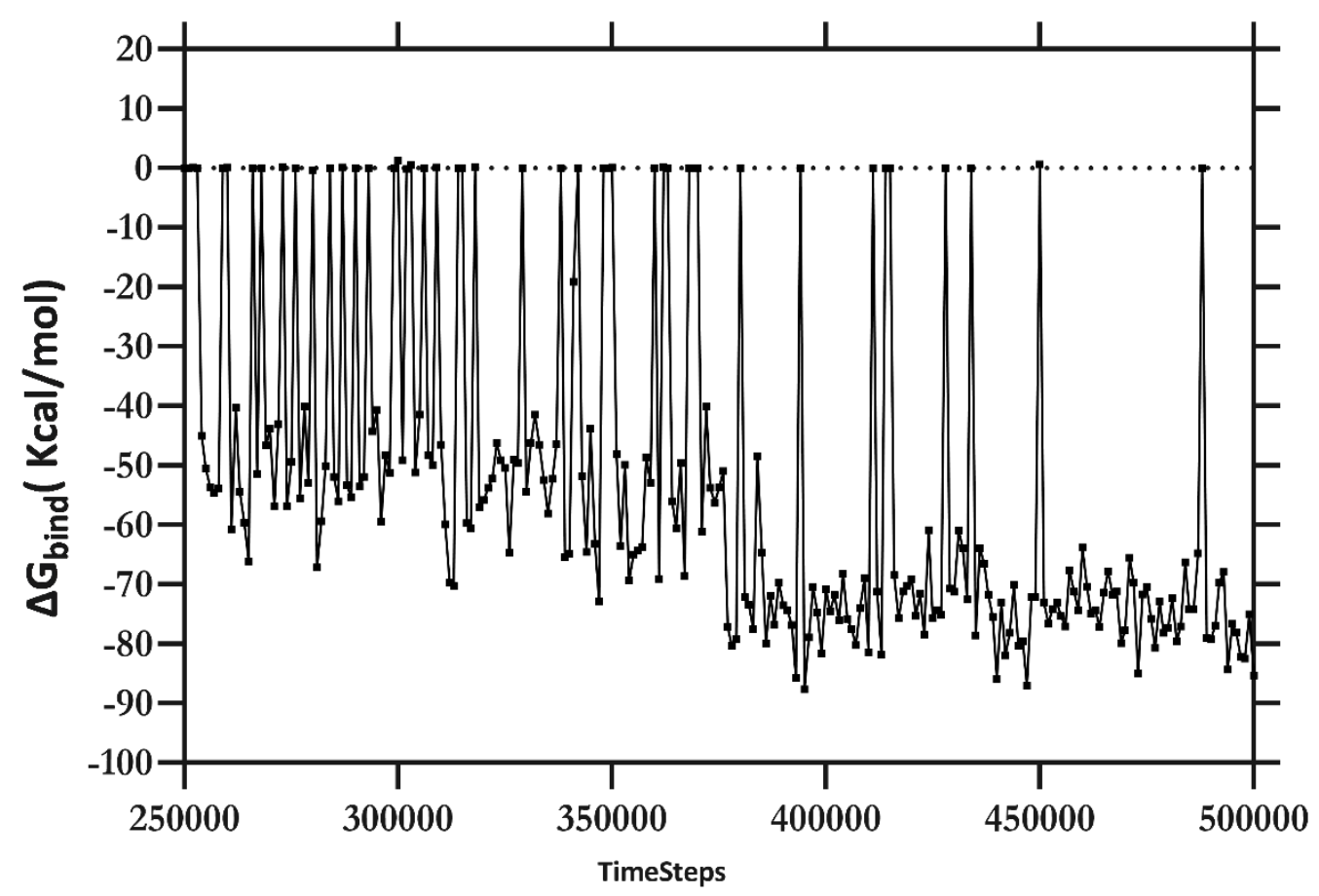


Figure 8

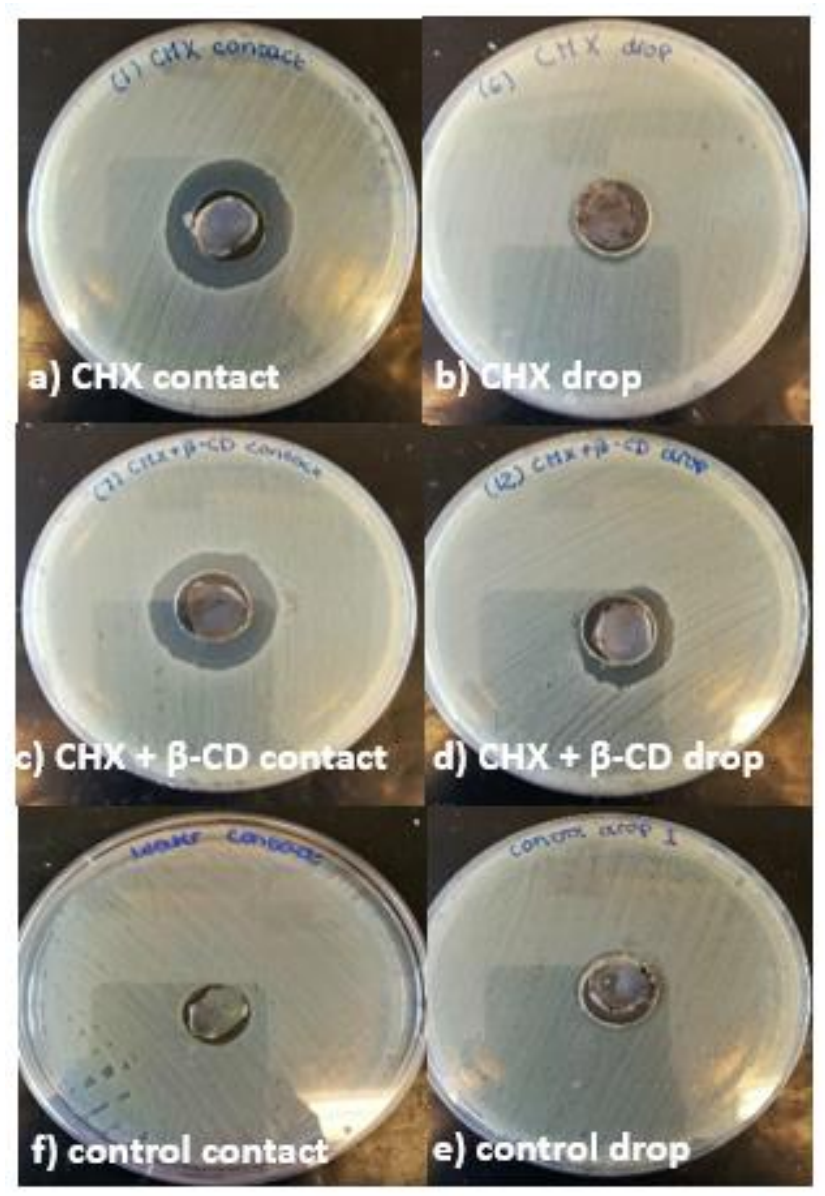


Figure 9

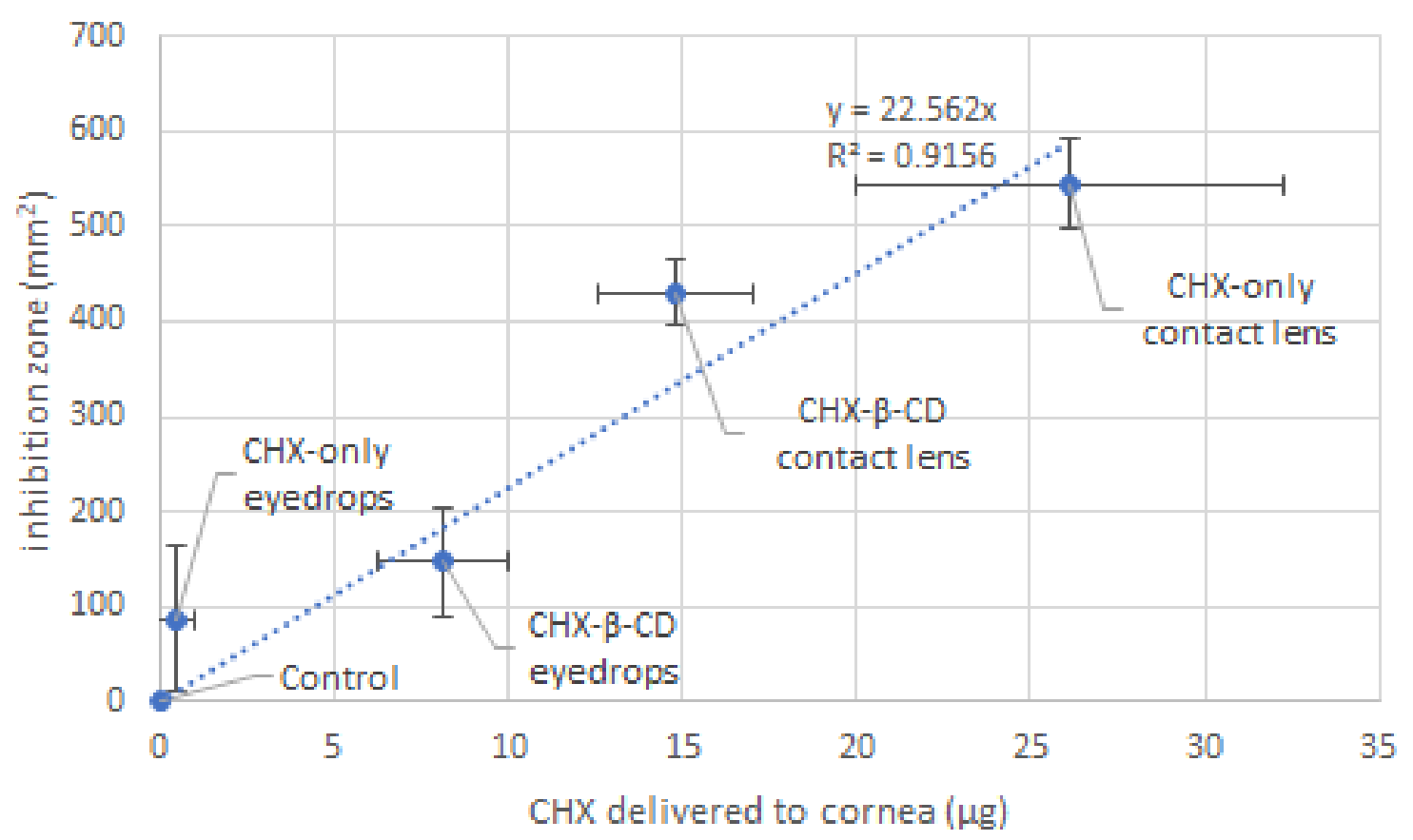


Figure 10

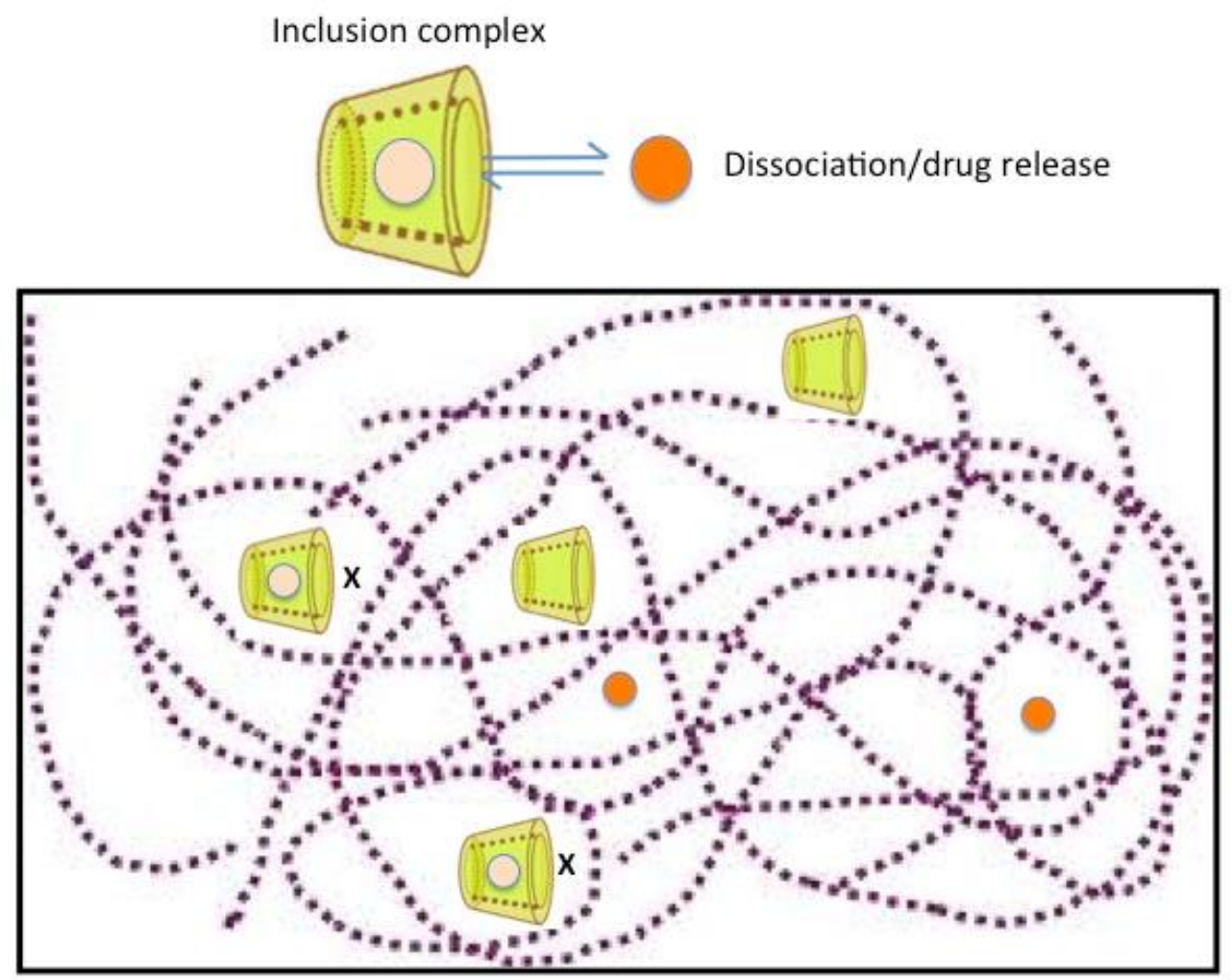




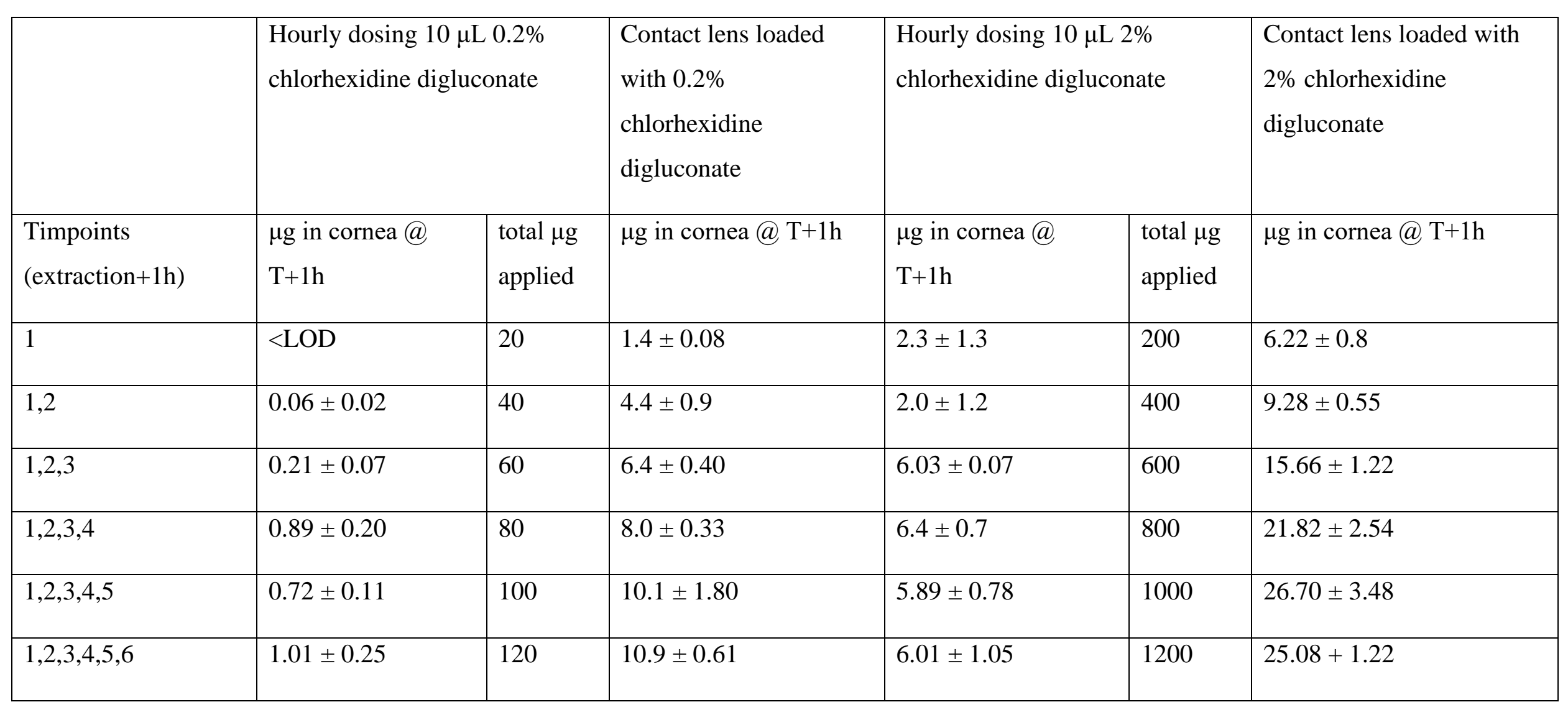

Table 1 The amount of chlorhexidine localised in porcine corneas as a function of dosing frequency, 1 hour after last dose, at concentrations of 0.2 and $2 \%(n=3 \pm S D)$. Simulated tear fluid applied throughout. 


\begin{tabular}{|l|c|}
\hline Delivery method & $\begin{array}{c}\text { Average Zone of } \\
\text { Inhibition }(\mathbf{m m})\end{array}$ \\
\hline Chlorhexidine-only contact lens & $30.3 \pm 1.0$ \\
\hline Chlorhexidine- $\beta-C D$ contact lens & $27.8 \pm 0.8$ \\
\hline Control contact lens & 0 \\
\hline Chlorhexidine-only eyedrops & $18.3 \pm 2.9$ \\
\hline Chlorhexidine- $\beta$-CD eyedrops & $20.3 \pm 1.9$ \\
\hline Control eyedrops & 0 \\
\hline
\end{tabular}

Table 2 Average zone of inhibition (mm) against E. coli from chlorhexidine containing corneas, according to the chlorhexidine delivery method.

Chlorhexidine was delivered to corneas by either chlorhexidine-only eyedrops, chlorhexidine- $\beta$-CD eyedrops, chlorhexidine-only loaded contact lens or chlorhexidine- $\beta-C D$ loaded contact lens while irrigated with STF. Six hours after initiation of treatment, corneas were excised and placed in wells for the well diffusion assay. 